\title{
1 The RNA binding protein DAZL functions as repressor \\ 2 and activator of maternal mRNA translation during oocyte maturation
}

4 Cai-Rong Yang1,2,3, Gabriel Rajkovic ${ }^{1,2,3}$, Enrico Maria Daldello ${ }^{1,2,3}$, Xuan G. Luong ${ }^{1,2,3}$, 5

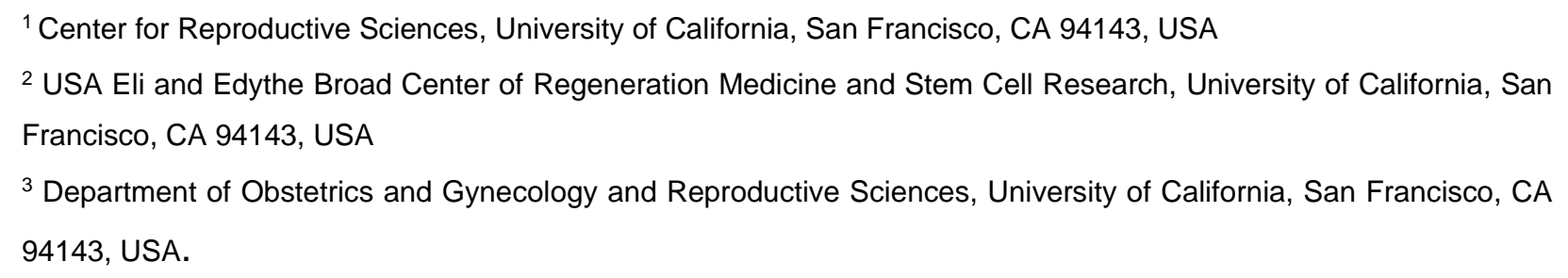

Address for correspondence:

Marco Conti

513 Parnassus Ave, Box 0556

San Francisco, CA 94143

Phone: (415)476-2695

FAX (415) 502-7866

Email: contim@obgyn.ucsf.edu

DAZL, maternal mRNA, translation, activator, repressor 


\section{Abstract}

29 Deleted in azoospermia like (DAZL) is an RNA-binding protein playing critical function during gamete development. In fully-grown oocytes, DAZL protein is detected in prophase and levels increase four to five fold during reentry into the meiotic cell cycle. Here, we have investigated the functional significance of this DAZL accumulation in maturing oocytes. Oocyte depletion of DAZL prevents progression to MII. This maturation block is associated with widespread disruption in the pattern of maternal transcripts loading on ribosomes and their translation measured using a RiboTag IP/RNASeq or qPCR strategy. In addition to decreased ribosome loading of a subset of transcripts, we found that DAZL depletion causes also translational activation of distinct subset of mRNAs. DAZL binds to mRNAs whose translation is both repressed and activated during oocyte maturation. Unexpectedly, DAZL depletion also causes increased ribosome loading of a subset of mRNAs in quiescent GV-arrested oocytes. This dual role of repression and activation is recapitulated by using YFP reporters including the 3'UTR of DAZL targets. Injection of recombinant DAZL protein in DAZL-depleted oocytes rescues the translation of these targets as

42 well as maturation to MII. Mutagenesis of putative DAZL-binding sites in these candidate mRNAs

43 mimics the effect of DAZL depletion. These findings demonstrate that DAZL regulates translation

44 of maternal mRNAs in mature oocytes, functioning both as translational repressor and activator. 


\section{Introduction}

48 In both males and females of most species, production of gametes is a developmental process 49 that spans embryonic, fetal, and postnatal life and is essential for the transfer of genetic information to the progeny ${ }^{1}$. Germline lineage specification, expansion of the gamete precursors (PGCs), meiosis, and terminal differentiation into functional gametes are all elaborate processes that require extensive regulation of gene expression ${ }^{1,2}$. Together with unique transcriptional and epigenetic mechanisms, regulation of translation plays a critical role in differentiation of these germ cells ${ }^{3,4,5}$.

\section{5}

In a mature mRNA, several domains play a critical role in the regulation of translational efficiency and stability ${ }^{6}$. These include the CAP region of the mRNA, the 5' and 3' UTR, and the poly(A) tail ${ }^{6}$, 7,8 . Translation of an mRNA is modulated through the interaction of numerous proteins with these domains of a mRNA. Indeed the assembly of ribonucleoprotein (RNPs) modulates every aspect of mRNA translation and stability. A subgroup of RNA-binding protein (RBPs) interacts with the 3'UTR of an mRNA. These RBPs participate in the formation of RNPs that are critical for the control of translational efficiency, stability and localization of the mRNAs ${ }^{9}$. These properties place these RBPs in a critical role in the control of protein synthesis. Among the several RBPs uniquely expressed in the germ line is the family of the Daz RNA-binding proteins ${ }^{10,11}$. DAZ, DAZL and BOULE are germ-cell specific RBPs essential for gametogenesis from worms to humans ${ }^{11}$.

DAZL KO prevents differentiation of PGCs ${ }^{10,12,13}$. It has been proposed that DAZL functions as a translational activator by recruiting poly $(A)$ binding proteins, which in turn promotes and stabilize interaction with the cap of mRNA, a loop conformation thought to promote stability and translational efficiency ${ }^{14}$. However, additional studies in PGCs suggest a repressive function for this RBP in the control of zebrafish embryogenesis and in the mouse ${ }^{15}, 16$. Moreover, depletion of DAZL during spermatogenesis has been associated with mRNA destabilization ${ }^{17}$.

We have previously reported that acute depletion of DAZL from fully grown mouse oocytes using morpholino oligonucleotides causes disruption of the progression through meiosis ${ }^{18,19}$. Here we have used this in vitro model to define the pattern of translation dependent on the function of this RBP. We find that DAZL depletion causes both increases and decreases in translational efficiency of a wide range of transcripts expressed in the oocyte and these effects are reversible and recapitulated by regulation of reporter translation of candidate DAZL targets. 


\section{Results}

DAZL is expressed in fully grown oocytes and is depleted upon inhibition of DAZL mRNA translation

81 We have reported that DAZL protein is detectable in fully grown GV-arrested oocytes with protein 82 levels increasing further up to $\mathrm{MII}{ }^{18}$. This finding is at odd with data of others where DAZL protein was only borderline detectable or could not be detected ${ }^{20,21}$. Here, we have re-evaluated the expression of DAZL during oocyte maturation using a newly developed monoclonal DAZL antibody (see 'Materials and Methods'). Western blot analysis of extracts from oocytes at different stages of maturation $(0,2,4,6$, 8hrs) reveals an immunoreactive polypeptide with mobility corresponding to that of DAZL (37 kDa) in GV oocytes, and a three-to-fourfold increase in protein levels up to $8 \mathrm{hrs}$ of in vitro maturation (MI) (Fig.1a, Supplenmentary Fig. 1e), in complete agreement with our previous reports using in vivo matured oocytes. Note that the identity of the immunoreactive band is further confirmed by morpholino kncockdown experiments (see Fig.1b, Supplementary Fig. 1f). Thus, two different antibodies document that DAZL is expressed in GV oocytes and that accumulation of the protein increases with maturation, conclusions consistent with our Dazl mRNA translation data with in vivo and in vitro matured oocytes (Supplementary Fig.1a, b, c).

To determine whether preventing Dazl mRNA translation effectively depletes the oocytes of the DAZL protein, GV-arrested oocytes from RiboTag ${ }^{\mathrm{fl} / \mathrm{fl}}$ : Zp3-CRE, Dazl ${ }^{+/+}$or Dazl ${ }^{+/-}$mice were injected with a scrambled (Con-MO) or DAZL targeting morpholino (DAZL-MO) respectively, to maximize DAZL protein removal. Blockage of DazlmRNA translation by this specific MO markedly reduces $(94.235 \%$ decrease $+/-0.025$, Mean $+/-$ SEM, $N=3$ ) the endogenous DAZL protein expression compared to a CON-MO (Fig.1b). To further assess the effectiveness and specificity of the treatment, we used RiboTag IP/qPCR to evaluate the MO effect on ribosome loading onto endogenous mRNAs during the transition from germinal vesicle stage (GV) to Meiosis I stage (MI). We observe a significant decrease in ribosome recruitment onto the Daz/ mRNA, confirming the effectiveness of the $\mathrm{MO}$ in blocking initiation of translation, with consequent depletion of the protein from oocytes (Fig. 1c). TEX19.1 is an established target of DAZL ${ }^{19}$. We show that the Tex19.1 mRNA loading on ribosomes is significantly decreased after injection of DAZL-MO at the MI stage (Fig. 1d). Conversely, knockdown of DAZL had no effect on ribosome loading onto the non-target CcnB1 (Fig. 1e), which is again consistent with our previous report ${ }^{18,19}$. No detectable effect on total transcript levels was detected under these conditions. Confirming what previously 
110

111

112

113

114

115

116

117

118

119

120

121

122

123

124

125

126

127

128

129

130

131

132

133

134

135

136

137

138

139

140

141

142

reported by us, DAZL depletion disrupts oocyte maturation to MII (see below). Further control experiments where immunoprecipitation was performed with WT rather than RiboTag mice yield only background signal, confirming the specificity of the RiboTag immunoprecipitation (Supplementary Fig.1d). These pilot experiments document that DAZL knockdown specifically disrupts DAZL target loading onto ribosomes with high selectivity since it does not affect the translation of CcnB1, an mRNA that does not interact with DAZL. Additionally, they confirm that RiboTag IP in oocytes depleted of DAZL is an effective strategy to assess the role of this RBP in endogenous maternal mRNA translation.

\section{Ribosome loading onto maternal mRNAs is disrupted in oocytes depleted of DAZL}

For a genome-wide analysis of the effect of DAZL depletion on translation of oocyte endogenous mRNAs, GV oocytes from RiboTag ${ }^{\mathrm{f} / / f l}:$ Zp3-CRE, Dazl ${ }^{+/+}$or Dazl ${ }^{+/-}$mice were injected with ConMO or DAZL-MO. After overnight recovery, oocytes were collected at $0 \mathrm{hr}$ (GV) or cultured in inhibitor-free medium to mature for up to $6 \mathrm{hrs}$ (MI). Although the changes in translation would be more pronounced if measured in fully matured MII oocytes, this short maturation time was selected to monitor early effects of DAZL depletion, thus, avoiding the potential confounding effects of the blockage of maturation to MII, and a potential decrease in oocyte viability. When we compare total mRNAs from CON-MO and DAZL-MO in GV-arrested oocytes (overnight incubation in PDE inhibitors), few differences are detected (Fig. 2a). Also comparison of ribosome loading in the $\mathrm{CON}-\mathrm{MO}$ at $0 \mathrm{hr}$ and $6 \mathrm{hrs}$ shows changes in ribosome loading qualitative similar to those reported previously with polysome array or other RiboTag IP/RNASeq data sets with non injected oocytes (Supplementary Fig. 2a). However, when we compare RiboTag IP/RNASeq in the 6 hrs DAZL-MO versus 6hrs CON-MO group, we observe complex changes in maternal mRNA ribosome loading (Fig. 2b). Ribosome loading onto the majority of transcripts present in the oocyte is not significantly affected by the DAZL depletion (grey dots in Fig. 2b). However, we detect a decrease in ribosome loading onto a group of transcripts (blue dots in Fig. 2b, 551 transcripts, FDR $<0.05$ ), a finding consistent with the theory that DAZL functions as a translational activator. Surprisingly, we also identify a group of transcripts whose translation increases (red dots in Fig. 2b, 170 transcripts, FDR < 0.05) after DAZL removal. This latter finding indicates that directly or indirectly DAZL has a role in repression of translation. As an example of the RNASeq data, ribosome loading onto Tex19.1, Txnip, Akap10, and Nsf mRNAs at $0 \mathrm{hr}$ and $6 \mathrm{hrs}$ of maturation are reported in Fig. $2 d, 2 e$. These mRNAs are significantly immunoprecipitated by DAZL antibody as shown in our DAZL RIP-Chip dataset (Fig. 2c). We found no clear pattern in the changes in total mRNA levels after DAZL depletion (Fig. 2d). RiboTag IP/RNA-Seq shows an increase in 
ribosome loading (HA immunoprecipitation) for transcripts Tex19.1 and Txnip during maturation in CON-MO injected oocytes, whereas the recruitment is blunted after DAZL KD in MI stage(Fig. 2e). Conversely, ribosome loading onto Akap10 and Nsf mRNAs is increased after DAZL depletion (Fig. 2e). These data provide an initial indication that that DAZL functions not only a translational activator, but also a translational repressor during the GV-to-MI transition. Given the fact that no significant differences in total mRNA were detected between the CON-MO and DAZLMO groups, calculation of the translational efficiency (HA-IP:input ratio) shows the same trend (Supplementary Fig. 2b).

\section{The dual effect of DAZL depletion is confirmed by RiboTag IP/qPCR}

To confirm the opposing effect of DAZL depletion on translation, we performed RiboTag IP/qPCR with independent biological samples to monitor the recruitment of representative transcripts to the ribosome/translation pool. GV stage oocytes from wild type or DAZL Heterozygous mice were injected with control or DAZL MO. After overnight preincubation with $2 \mu \mathrm{M}$ milrinone, oocytes were cultured in inhibitor-free medium. Oocytes were collected at 6 hrs for RiboTag IP /qPCR analysis. This RNA quantification confirms that the overall transcripts levels (input of RiboTag IP/qPCR) are not affected, including transcripts coding for Dazl, Tex19.1, Txnip, Rad51C, Btg4, Ero1, Oosp1, Obox5, Ireb2, and Tcl1 (Supplementary Fig. 3a). However, RiboTag IP/qPCR shows a decrease translation for several candiates after DAZL removal, similar to that observed with the RiboTag IP/RNASeq (Fig. 3a, c). Dppa3 and CcnB1 are used as negative control, as they are not regulated by DAZL during oocyte maturation ${ }^{18,19}$. Conversely, translation of transcripts coding for Akap10, Cenpe, Nsf, Ywhaz, Nin, and YTHDF3 is upregulated after DAZL removal (Fig. 3b, d), while the overall transcripts levels are not changed (Supplementary Fig. 3b). Gdf9, used as negative control, is not affected by DAZL depletion. These results not only confirm our RiboTag IP/RNASeq data, but also indicate that DAZL may plays dual function (both translational activator and repressor) during oocyte maturation.

\section{DAZL physically interacts with transcripts whose translation is upregulated or downregulated after DAZL removal}

The above findings open the possibility that DAZL binding to maternal mRNA leads to both increase and decrease in translation. If these were correct, DAZL should bind to maternal mRNAs whose translation is upregulated or downregulated during oocyte maturation. To test this hypothesis, we analyzed a previously generated DAZL RIP-Chip dataset. In this DAZL RIP-Chip dataset, 811 transcripts are significantly immunoprecipitated ( $>1.5$ fold enrichment as compared 
175

176

177

178

179

180

181

182

183

184

185

186

187

188

189

190

191

192

193

194

195

196

197

198

199

200

201

202

203

204

205

206

to $\lg$ ) by DAZL antibodies during the GV to MII transition. A scatter plot (Fig. 4a) of these data shows DAZL binding to both upregulated transcripts and downregulated transcripts, a finding consistent with the two classes distribution of the ribosome loading transcripts (Supplementary Fig 2 a). This analysis is again consistent with the hypothesis that DAZL interacts with both classes of transcripts whose translation may increase or decrease during oocyte maturation. A more quantitative comparison of the mRNA immunoprecipitated by DAZL antibody and the transcripts whose translation is affected by DAZL depletion shows that 212 downregulated and 49 upregulated (total 251) transcripts are also immunoprecipitted by DAZL antibodies. A sizable number of transcripts (215) are not affected or changes do not reach statistical significance (Supplementary Fig. 4).

We wished to next confirm that transcripts whose translation increases after DAZL depletion are indeed direct target of DAZL. Because no sufficient signal could be obtained in DAZL RIP when using 200 oocytes with currently available antibodies, we used mouse embryonic stem cells(ES) for DAZL RIP. It is established that ES cells in the ground state express DAZL as well as a large number of two-cell embryo transcripts, mRNAs often expressed also in oocytes ${ }^{15,22} 23$. ES cells were cultured in DMEM medium $+2 i$ and collected for DAZL IP/qPCR analysis. The results are normalized to the IgG IP. This DAZL RIP experiment in ES cells shows that transcripts of Dazl, Tex19.1, Txnip, Rad51C, Btg4, Ero1lb, Ireb2, and Tcl1b (Fig. 4B, red), whose translation is downregulated by DAZL removal, and transcripts of Akap10, Nsf, Ywhaz, Nin, and YTHDF3, whose translation is upregulated by DAZL removal (Fig. 4b, blue), are all specifically immunoprecipitated by DAZL antibody (Fig. 4b) . However, Cenpe, a transcript whose translation is upregulated by DAZL removal, could not be immunoprecipitated by the DAZL antibody, suggesting that DAZL may also act as a translational repressor through an indirect pathway. Nevertheless, most of the transcripts whose translation increases/decreases after DAZL depletion are directly interacting with DAZL in oocytes and ES cells.

\section{The 3' UTR of representative transcripts Oosp1 and Obox5 recapitulates the effect} of DAZL depletion on translational activation.

Oosp1 and Obox5 are two oocyte-specific transcripts whose translation is affected by DAZL removal as determined in both the RiboTag IP/RNA-Seq dataset and in the RiboTag IP/qPCR validation. OOSP1 (oocyte secreted protein 1) was initially identified as a novel oocyte-secreted protein ${ }^{24}$. OBOX5 (oocyte specific homeobox 5) is a member of the Obox family of proteins but its function is unclear ${ }^{25}$. These mRNAs were chosen because of their robust translational 
activation in meiosis and their relatively simple 3'UTR. To verify the effect of DAZL on translation of these two candidate mRNAs, a YFP reporter was fused to the Oosp1 or Obox5 3'UTR and these constructs were injected in oocytes together with either Con MO or DAZL MO. A fully polyadenylated $m C h e r r y$ reporter was used as a control of the volume injected. The accumulation of YFP and mCherry in individual oocytes was recorded throughout meiotic maturation and YFP signals were corrected by the level of co-injected mCherry signal. Data are expressed as changes over $0 \mathrm{hr}$ (GV stage), as differences in reporter accumulation were detected in GV-arrested oocytes (see below). By measuring the average YFP signals throughout maturation, the accumulation of YFP-Oosp1 and YFP-Obox5 reporter in CON-MO group closely follows the corresponding ribosome loading onto the endogenous mRNA; however, DAZL depletion causes at least $50 \%$ decrease in translation in Oosp1 and Obox5 reporter during oocyte maturaion (Fig. $5 \mathrm{a}, \mathrm{c})$. We further assessed the rates of translation during oocyte maturation of the two reporters by fitting the YFP/mCherry data during GV (0-2 hrs) and after GVBD (4-8 hrs) (Fig. 5b, d). We found a significantly decrease of Oosp1 $(p<0.0001)$ and Obox5 $(p<0.0001)$ translation rates in DAZL MO injected oocytes (Fig. 5b, d), confirming that DAZL depletion decreases the translation of these reporters during oocyte maturation. Consistent with our RiboTag IP/RNASeq data and translational efficiency of Oosp1 and Obox5 is affected by DAZL depletion (Supplementary Fig. 5). Ccnb1 3'UTR co-injected with either CON-MO or DAZL-MO shows no obvious changes in translational accumulation between the two groups, confirming the selective effect of the DAZL depletion (Fig. 5e, f).

To confirm that the depletion of DAZL protein with the specific MO is the sole cause of the decreased translation of the reporter, we performed the following rescue experiment. A human recombinant DAZL protein was injected together with the DAZL MO and the Oosp1 reporter. As observed above, DAZL depletion causes a decrease in the rate of translation of the Oosp1 reporter. This decrease is completely rescued when the recombinant DAZL protein is coinjected with the DAZL MO (Fig. 6a, b). The rescue effect of the DAZL protein was not limited to the translation efficiency. As previously reported, DAZL depletion on a het background almost completely prevents oocyte maturation to MII (Control MO 69.7\% vs DAZL MO:7\%). Conversely, when the DAZL MO is co-injected with a recombinant DAZL protein, oocytes complete maturation to $\mathrm{MII}$ at a rate (63\%) similar to control injected oocytes(Fig. 6c). Taken together with the RiboTag IP / RNA-Seq and qPCR data (Fig. 2, Fig. 3), the reporter measurements further support the conclusion that the DAZL protein plays a role in the translational activation of these two target 
mRNAs, that their 3'UTR mediates the effect of this RBP on translation, and that DAZL depletion is the cause of decreased translation.

DAZL depletion increases the translation of Oosp1 and Obox5 mRNAs in GVarrested oocytes

In the above experiments on reporter translation, we consistently observed that translation of both reporters during the first two hours of incubation, when the oocytes are still GV-arrested, is significantly increased in the DAZL-MO injected group (Oosp1: $p<0.0001$ and Obox5 $p=0.0007$ ) (Fig. 7b, d). To verify this apparent de-repression in DAZL-depleted, quiescent oocytes, we reanalyzed the RiboTag IP/RNASeq data sets. We found that ribosome loading on both Obox5 and Oosp1 during GV-arrested is also increased in this dataset (Supplementary Fig. 6). To remove any possible bias due to variation in the total $\mathrm{mRNA}$, we next calculated the translational efficiency (TE) of these two transcripts after DAZL depletion. Indeed, the TEs for Oosp1 and Obox5 are significantly increased in GV oocytes depleted of DAZL (Fig. 7a, b), whereas the CcnB1 translation is not affected (Fig. 7e) To assess whether this effect of DAZL depletion is widspread, we reanalyzed the RiboTag IP RNASeq data and found that ribosome loading of approximately 70 transcripts is significantly increased after DAZL removal in GV oocytes (69, Supplementary Fig. 6 red, FDR<0.05). This latter finding provides further evidence for a repressive role of DAZL prior to meiotic resumption.

Efficient translation of Oosp1 and Obox5 reporters requires the presence of DAZL

\section{binding element.}

259 Collectively, the above experiments strongly indicate a translational regulation of the Oosp1 and 260 Obox5 transcripts are affected by DAZL MO injection during GV to MI transition. To test whether 261 this effect is due to binding of DAZL to these target mRNAs, we mutated the consensus putative DAZL-binding sites (UU[G/C]UU) by replacing critical nucleotides with adenosine in Oosp1 3'UTR or Obox5 3'UTR. We have previously shown that this mutations disrupts DAZL binding ${ }^{19,26 . ~ A ~}$ schematic representation of Oosp1 and Obox5 3'UTR with mutant DAZL binding sequence is reported in Fig. 8a. When YFP reporter tagged with mutant Oosp1 3'UTR or Obox5 3'UTR YFP reporters were injected in oocytes and their rate of translation were monitored during maturation, mutation of a single DAZL-binding site in either Oosp1 or Obox53'UTR is sufficient to significantly decrease the rate of reporter accumulation (Fig. 8c, d, f, g) during meiotic resumption. Of note, translation in GV stage oocyte as compared to wildtype reporters. This increased rate of 
271 translation of the reporter was confirmed in a different experimental paradigm where control and

272 DAZL depleted oocytes are maintained in GV stage. Also under these conditions, the translation

273 rate of the Oosp1 reporter is increased, whereas the CcnB1 mRNA is not affected (Supplementary

274 Fig. 7) This latter finding is consistent with the results of DAZL MO of the RiboTag IP/RNASeq

275 experiment (Fig. 7a, c), confirming that DAZL functions as translational repressor also in GV-

276 arrested oocyte.

\section{Discussion}

278 With the experiments described above, we provide evidence that the RNA-binding protein DAZL

279 plays a function in fully-grown oocytes by shaping the pattern of maternal mRNA translation at

280 this critical transition of gametogenesis. Our data document that DAZL has both inhibitory, and

281 stimulatory, effects on translation in quiescent oocytes as well as during meiotic resumption. This

282 dual function parallels that of CPEB1, which is considered to be the master regulator of translation

283 in oocytes, and reinforces the concept that DAZL cooperates with CPEB to repress and active

284 translation of maternal mRNAs. Given the finding that DAZL-MO treatment prevents progression

285 through meiosis, it is proposed that the DAZL function is essential for oocyte maturation.

286 Several lines of evidence support the conclusion that DAZL is expressed and functional at the 287 end of oogenesis in fully grown mouse oocytes. The mRNA coding for DAZL is expressed at high 288 levels in fully-grown MII oocytes ${ }^{18}$. RiboTag IP/RNASeq data confirmed by qPCR document that 289 the Dazl mRNA is actively translated and translation increases during oocyte maturation. In line 290 with mRNA ribosome loading, DAZL protein is detected by Western blot with two distinct 291 antibodies. The progressive increase in the DAZL protein during maturation is consistent with the 292 increase in translation, further strengthening the view that this RBP accumulates during oocyte 293 maturation. Finally, the RIP-Chip data document that the DAZL is actively interacting with 294 hundreds of maternal mRNAs. All these independent pieces of evidence strongly support the 295 hypothesis of expression and function if this RBP at the final stage of oogenesis.

296 The function of DAZL in translation is further confirmed by a loss-of-function approach. Morpholino 297 oligonucleotide interference with mRNA translation was used on a DAZL-heterozygous 298 background, since homozygous deletion of this gene precludes oocyte development. As detailed 299 above, we chose to determine the effect of DAZL effect on translation in MI to avoid secondary 300 effects due to the block in oocyte maturation. Injection of DAZL-MO interrupts Dazl mRNA 301 translation as detected by RiboTag IP/qPCR. In parallel with decreased translation, Wester blot 302 analysis of oocyte extracts shows a reproducible decrease of more than $90 \%$ in DAZL protein. 
The specificity of this treatment is supported by the data showing that ribosome loading onto the mRNAs for CcnB1, Dppa3 and Gdf9 is not affected. At the transcriptome level, the overnight incubation to deplete the DAZL-MO injected oocytes has minimal effect on total transcript levels, arguing against a generalized disruption of the oocyte viability. All these findings increase confidence that the KD of DAZL is effective in depleting the oocytes of the DAZL protein and that its effect is specific.

The analysis of the translatome in DAZL-depleted oocytes indicates that approximately 800 maternal mRNAs show altered on the level of ribosome loading. Together with the DAZL RIPChip data, this analysis confirms the presence of a large number of maternal mRNA targets for this RBP. Tex19.1, Txnip, Rad51C, Btg4, Oosp1, Obox5, Ireb2, andTcl1 are examples of the more than 200 mRNAs regulated by DAZL on the basis of the decreased ribosome loading after DAZL depletion and the observation that these mRNAs are immunoprecipitated by DAZL antibodies. These findings confirm our and others previous observations that Tex19.1 mRNA is a DAZL target ${ }^{19,27}$. TEX19.1 protein accumulates during oocyte maturation and data in the testis indicate that this protein may be involved in the regulation of transposon expression ${ }^{28,29,30 .}$ Similarly, DAZL regulation of Btg4 mRNA translation has been reported by others ${ }^{31,32,33}$. In both mouse and human, Btg4 transcripts are highly enriched in the ovary and testis. A consequence

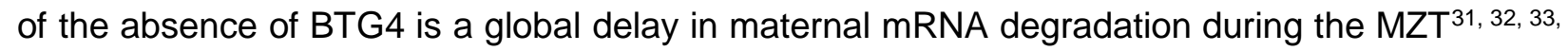
34. Given the involvement of BTG4 in mRNA destabilization, one would expect that DAZL depletion would induce mRNA stabilization by preventing Btg4 accumulation. However in our experimental paradigm, DAZL depletion and consequent decreases in BTG4 accumulation at $6 \mathrm{hrs}$ are probably not sufficient to produce detectable effect on mRNA stabilization. mRNA destabilization is detected at $8 \mathrm{hrs}$ of oocyte maturation (data not shown). Finally, it should be noted that ribosome loading onto all the above listed mRNAs increases during oocyte maturation and most of the mRNAs also contain at least one putative CPE element in the 3'UTR. Thus, one function of DAZL is likely to increase the translation of these mRNA during maturation, a function likely synergistic with that of CPEB, as we have described for Tex $19.1^{19}$. This conclusion is supported by the reporter translation of YFP-Oosp1 and YFP-Obox5. Similarly, the data with the YFPCenpE reporter (Supplementary Fig. 8) are consistent with the view that a group of transcripts, that include Akap10, Cenpe, Nsf, Ywhaz, Nin, and YTHDF3, are translationally repressed by DAZL.

Our DAZL RIP-Chip data indicates that DAZL interacts with approximately $800 \mathrm{mRNAs}$ expressed in the oocytes. The interaction of several candidate mRNAs with DAZL was also confirmed in ES 
cell extracts. Data are available for Dazl mRNA targets in the fetal gonad of the mouse and human. Of the mRNA shown to interact with DAZL in human fetal ovary ${ }^{35}$, Sycp1 and Tex11 are not detected in GV oocytes and the ribosome loading of Smc1b although decreased in our data set does not reach statistical significance. A comparison between the human fetal gonad data ${ }^{35}$ and our mouse RIP data shows overlap in immunoprecipitation of 72 mRNAs including Trip13, Rad 51, Spin1, Kit, and Arpp19.

One consistent finding is that Dazl mRNA is immunoprecipitated with DAZL antibody in both mouse and human fetal ovary. This finding reinforces the idea that DAZL is involved in an aoutregulatory loop controlling its own translation ${ }^{18}$.

Of the mRNAs identified in the DAZL RIP-Chip, 261 of 476 transcripts are affected by DAZL depletion (Supplementary Fig. 4). The limited overlap between the transcripts recombined in the RIP and in the RiboTag IP/RNASeq is in part due to the differences in annotation of the two platforms (300 genes not shared) or to the different filtering of the data. Our numbers are also considerably lower than those reported by Zagore et al. using HITS-CLIP with testis extracts ${ }^{17}$. This latter difference may be due to the fact that we did not use crosslinking for our experiment but also to the fact that very low amounts of cell protein is available from the oocyte. Thus, the affinity of the antibody for DAZL becomes limiting. Similar to the finding of Zagore et al. ${ }^{17}$, we find that about 200 mRNAs that bind DAZL are not affected by DAZL depletion. This discrepancy again can be due to filtering of the data and the cutoffs imposed. Also, we should point out that in our experimental paradigm, we are measuring acute effects of DAZL depletion and if longer incubation times are used the number of mRNAs affected would increase substantially.

A previous report in the testis proposes that DAZL is involved in mRNA stabilization ${ }^{17,36,37,38 .}$ Therefore it is possible that DAZL depletion affects translation indirectly by destabilizing mRNAs. However, overnight depletion of DAZL has minimal effects on the oocyte transcriptome, lessening the possibility of destabilizing effects on maternal mRNAs. Moreover, all the data of the candidates we more thoroughly investigated are inconsistent with the destabilization hypothesis, as we cannot detect a decrease in total mRNA. Therefore, the decreased translation for these candidate DAZL targets is not due to destabilization of these mRNAs. Moreover, many of the effects of DAZL depletion on translation continue to be present when the TE is calculated, indicating that mRNA stabilization is not a factor in ribosome loading. However, the timeframe of our experiments is considerably short and we cannot exclude that longer time causes of DAZL depletion uncovers effects of DAZL depletion on mRNA stability. 
Recently, it has been reported that DAZL is dispensable for oocyte maturation, but that instead its overexpression has deleterious effects on oocyte developmental competence ${ }^{20}$. This conclusion is based on the observation that DAZL protein is markedly decreased in adult ovary in comparison with neonatal ovary; however, the variable ratio somatic:germ cells in the gonad during development may account, at least in part, for these differences ${ }^{20}$. Our data on DAZL protein expression detected with two distinct antibodies, the RIP-Chip data, and the translational regulations described confirm the expression and increased accumulation of DAZL in the final stages of oocyte development. Genetic manipulations also led to the conclusion that the absence of DAZL does not produce overt phenotypes on oocyte maturation or fertility. The genetic background used in these experiments is a mixed background (ICR and C57BL/6N) while we use a pure $\mathrm{C} 57 \mathrm{BL} / 6$ background. It has been noted that the penetrance of the phenotypes associated with Dazl gene ablation are sensitive to the mouse background used ${ }^{12}$. However, the view that a DAZL needs to be expressed within a very narrow range of concentrations is consistent with our findings that DAZL has a dual effect on translation, functioning both as a repressor and activator. Therefore, it is possible that increased DAZL levels favors translational repression that would be detrimental to developmental competence. Aside from the genetic background of the mice used, not immediately evident is the explanation of why Dazl KO in neonatal oocytes produces no detectable phenotype on fertility. Possible off-target effects of DAZL morpholinos are inconsistent with the rescue experiments we have performed. In several cases, it has been observed that morpholino oligonucleotide treatment is associated with induction of $p 53^{39,40}$ (tp53 or trp53) or interferon response or toll like receptor ${ }^{41}$. Since transcription is repressed in GV oocytes, it is unlikely that $\mathrm{MO}$ off-target effects include changes in transcription. However, we noticed that trp53 mRNA becomes associated with ribosome during oocyte maturation and is immunoprecipitated with DAZL antibody. We could not detect clear effects of DAZL depletion on trp53 mRNA translation. Another possible explanation of the divergent observations is that the oocyte does not tolerate acute depletion of DAZL, while it has time to adjust to loss of DAZL during the follicle growth phase that starts in the neonate ovary. Genetic compensation has been shown to be at the basis of differences in phenotypes produced by mutations but not knockdowns ${ }^{42}$. Since we have shown that DAZL functions in partnership with $C P E B^{19}$, it is possible that this latter RBP would compensate for the loss of DAZL. In this respect, it would be important to determine whether CPEB expression is affected in the DAZL KO and how the translational program is executed in the absence of DAZL in the fully grown oocyte. 
400

401

402

403

404

405

406

407

408

409

410

411

412

413

414

415

416

417

418

419

420

421

422

423

424

425

426

427

428

429

430

0

Several scenarios may explain the dual repression/activation role of DAZL on translation. One possibility is that DAZL assembles different molecular complexes in GV-arrested and MI oocytes. DAZL has been shown to interact with PUM2 forming a translational repressor complex on the Ringo/Spy mRNA ${ }^{43}$. During maturation, the complex is dissociated leading to translational activation. Thus, one could envisage that DAZL is part of a repressive complex in mouse GVarrested oocytes and contributes to an activating complex in Ml stage oocytes. This scenario is reminiscent of the CPEB1 mode of action ${ }^{8,44}$. We have observed that the DAZL protein shifts in mobility on SDS/PAGE during oocyte maturation, suggesting that the protein becomes posttranslationally modified during maturation. Finally, it should be considered that the concentration of DAZL protein increases up to six fold during oocyte maturation. Thus, it may be possible that low loading on an mRNA is sufficient to repress translation, whereas loading of multiple DAZL proteins on a mRNA leads to activation of translation. Indeed, we and others have proposed that the number of loaded DAZL synergizes in activation of translation ${ }^{14,18}$.

In summary, our findings are consistent with a role of DAZL in the translation program executed during the final stages of oocyte maturation. The dual function as repressor and activator suggests that complex changes in the proteome in fully grown oocytes and during maturation are dependent on DAZL action. These findings imply that spontaneous DAZL mutations found in human may affect not only germ cell development in the fetal gonad but they also have an effect on oocyte quality. Such a possibility has been proposed with the description of missense mutations in infertile women ${ }^{45}$.

\section{Materials and Methods}

\section{Experimental animals}

All experimental procedures involving animal models used were approved by the Institutional Animal Care and Use Committee of the University of California at San Francisco (protocol AN101432). Pure C57BL/6 female mice (21-24 days old) carrying the DAZL TM1 ${ }^{\text {Hgu }}$ allele ( $\left.\triangle D A Z L\right)$ were generated as previously described ${ }^{18,19}$. Rpl22tm1.1Psam/J (RiboTag) mice, with a targeted mutation that provides conditional expression of the ribosomal protein L22 tagged with three copies of the HA epitope. Rpl22tm1.1Psam/J homozygous males were crossed with C57BL/6TgN (Zp3-cre) 82Knw (Jackson Laboratories) females to produce C57BL/6-Zp3creRpl22tm1.1Psam (Zp3cre-RiboTag) mice. For breeding Zp3RiboTagDazl ${ }^{++}$or Zp3RiboTagDazl+/- 
431

432

433

434

435

436

437

438

439

440

441

442

443

444

445

446

447

448

449

450

451

452

453

454

455

456

457

458

459

460

, C57BL/6-Zp3cre- RiboTag wild type or homozygous males were crossed with C57BL/6- RiboTag wild type or heterozygous $\triangle \mathrm{DAZL}$ females to obtain C57BL/6- $\mathrm{DAZL-ZP3cre-RiboTag} \mathrm{mice.}$

\section{Oocyte collection and microinjection}

Oocyte isolation and microinjection were performed using HEPES modified minimum essential medium Eagle (Sigma-Aldrich, M2645) supplemented with $0.23 \mathrm{mM}$ pyruvate, $75 \mu \mathrm{g} / \mathrm{mL}$ penicillin, $10 \mu \mathrm{g} / \mathrm{mL}$ streptomycin sulfate, and $3 \mathrm{mg} / \mathrm{mL}$ BSA, and buffered with $26 \mathrm{mM}$ sodium bicarbonate. To prevent meiosis resumption, $2 \mu \mathrm{M}$ cilostamide (Calbiochem, 231085) was added in the isolation medium. Oocyte in vitro maturation was performed using Eagle's minimum essential medium with Earle's salts (Gibco, 12561-056) supplemented with $0.23 \mathrm{mM}$ sodium pyruvate, $1 \%$ streptomycin sulfate and penicillin, and $3 \mathrm{mg} / \mathrm{mL}$ bovine serum albumin (BSA). For microinjection, cumulus cells were removed by mouth pipette from isolated cumulus oocyte complexes (COCs) and denuded oocyte were injected with $5-10 \mathrm{pL}$ of $12.5 \mathrm{ng} / \mu \mathrm{L}$ mRNA reporter using a FemtoJet Express programmable microinjector with an Automated Inverted Microscope System (Leica, DM4000B). After washing and pre-incubating overnight in a-MEM medium supplemented with 2 $\mu \mathrm{M}$ cilostamide, oocytes were released in inhibitor-free medium for in vitro maturation at $37^{\circ} \mathrm{C}$ under $5 \% \mathrm{CO}_{2}$.

\section{Oocyte morpholino antisense oligonucleotide microinjections}

Germinal vesicle (GV) stage oocytes were isolated from wild type (WT) or Dazl Heterozygous $\left(\right.$ Dazl $\left.^{+/}\right)$mice. After pre-incubated in $\alpha$-MEM medium supplemented with $2 \mu \mathrm{M}$ cilostamide for $1 \mathrm{hr}$ at $37^{\circ} \mathrm{C}$ under $5 \% \mathrm{CO} 2,5-10 \mathrm{pl}$ of $1 \mathrm{mM}$ morpholino oligonucleotides (Gene Tools) of standard control (5'-CCTCTTACCTCAGTTACAATTTATA-3') or against Dazl (5'CCTCAGAAGTTGTGGCAGACATGAT-3') were injected into WT or Dazl+/- oocytes using a FemtoJet express microinjector. Following overnight incubation in $\alpha-M E M$ containing $2 \mu \mathrm{M}$ cilostamide medium, oocytes were released in $\alpha$-MEM medium without inhibitor for in vitro maturation or recording under the microscope.

\section{RiboTag IP RNASeq}

Oocytes from RiboTag wild type and $\mathrm{Daz}^{+-}$mice were collected as described above. Wild type oocytes were injected with a CON-MO, while the Dazl ${ }^{+-}$oocytes were injected with a DAZL-MO. Control experiments show that Dazl ${ }^{+/-}$oocytes have maturation timing and PB extrusion rates identical to wild type oocytes but a 50\% decrease in Dazl mRNA and protein. In addition, pilot 
experiments showed a dosage effect in DAZL depletion and MII stage block when comparing DAZL MO injected in wild type oocytes versus DAZL-MO injected in Daz+/- oocyte.

Oocytes injected with Con-MO and DAZL-MO were precinubated overnight in the presence of 2 uM milrinone and the following morning transferred to maturation medium and incubated for 6 hrs. At the end of the incubation, only oocytes that had undergone GVBD were collected in $5 \mu l$ $0.1 \%$ polyvinylpyrrolidone (PVP) in PBS, flash frozen in liquid nitrogen, and stored at $-80^{\circ} \mathrm{C}$. In parallel, GV oocytes were kept in milrinone, then harvested and processed together with the MI oocytes. A total of 2000 oocytes ( $0 \mathrm{hr}$ and $6 \mathrm{hrs}$ with either CON-MO or DAZL-MO injection) were injected and cultured for the duplicate determination of the effect of DAZL depletion on ribosome loading of endogenous mRNAs. On the day when the RiboTag IP was performed, oocytes were thawed, lysed and an aliquot of the oocyte extract was saved and stored to measure total transcript levels before the IP. RiboTag IP was performed as described in the section on Immunoprecipitation. After IP, all samples were used for RNA extraction using the RNeasy Plus Micro kit (Qiagen, 74034). The quality of the extracted RNA was monitored with Bioanalyzer chips (Agilent). RNA samples were transferred to the Gladstone Institutes Genomics Core for cDNA library preparation using the Ovarion RNA-Seq System V2 (NuGen). Samples were sequenced using the HiSeq400 platform.

\section{Real-time $q P C R$}

Real-time qPCR was performed using Power SYBR PCR master mix with ABI 7900 Real-Time PCR system (Applied Biosystems). All oligonucleotide primers used in this project were designed against two exons flanking an intron to avoid amplification of genomic DNA (Supplementary Table 1). The specificity of each pair of primers was verified by using a unique dissociation curve, performed at the end of the amplification. Data was normalized to its corresponding input and lgG in RiboTag/qPCR for HA and DAZL antibody and expressed as the fold-enrichment of $2^{-\triangle \Delta C t}$.

\section{Western Blotting}

Oocytes were lysed in 10 $\mu \mathrm{l} 2 \mathrm{x}$ Lammli buffer (Bio-Rad) supplemented with mercaptoethanol, and a cocktail of phosphatase and protease inhibitors (Roche). The oocyte lysates were boiled for 5 mins at $95^{\circ} \mathrm{C}$ and then transferred to an ice slurry, then separated on $10 \%$ polyacrylamide gels and transferred to a polyvinylidene difluoride (PVDF) membrane. Membranes were blocked in 5\% milk for $1 \mathrm{hr}$ at room temperature and incubated with primary DAZL antibody (ab215718, Abcam, 1:1000) overnight at $4^{\circ} \mathrm{C}$. An antibody against $\alpha$-tubulin (T6074, Sigma-Aldrich; $\left.1: 1000\right)$ was used 
492

493

494

495

496

497

498

499

500

501

502

503

504

505

506

507

508

509

510

511

512

513

514

515

516

517

518

519

520

521

522

523

as a loading control. After overnight incubation, membrane was washed in TBS-Tween 20 (0.05\%) three times and incubated with HRP-conjugated secondary antibodies (Pierce; 1:5000) for $1 \mathrm{~h}$ at room temperature. The signals were detected using Super Signal West Fremto (Thermo Scientific, 34095).

\section{Culture of ES Cells}

ES cells were handled under sterile conditions and recovered in DMEM medium (high glucose, Glutamax, Pyruvate) at room temperature. After centrifuging the cells at $200 \mathrm{x} \mathrm{g}$ and resuspending with $1.5 \mathrm{ml}$ DMEM culture medium supplemented with $15 \% \mathrm{KOSR}, 2 \% \mathrm{FBS}$, 1\% 2-Mercaptoethanol, 1\% penicillin/streptomycin, 1\% MEM Non-Essential Amino Acids, 1000 U/ml LIF, $3 \mu \mathrm{M}$ CHIR-99021, and $1 \mu \mathrm{M}$ PD0325901, ES cells were cultured in multi-well plates coated with $0.1 \%$ gelatin in $\mathrm{H}_{2} \mathrm{O}$ and $\mathrm{FBS}$ at $5 \% \mathrm{CO}_{2}$ and $37^{\circ} \mathrm{C}$. Culture media was changed daily. When ES cells reached approximately $80 \%$ confluency, they were washed once with DPBS and incubating with $0.05 \%$ Trypsin for $1 \mathrm{~min}$. The reaction was stopped by adding ES cell culture medium without LIF and $2 \mathrm{i}$ and KOSR, KOSR has been replaced with $10 \%$ FBS in this medium (later called MEF Media). Cells were then pelleted by centrifuging at $200 \times \mathrm{g}$ for $5 \mathrm{~min}$. The ES cell pellet was dissolved in RNase-free PBS and stored at $-80^{\circ} \mathrm{C}$ for DAZL IP.

\section{Immunoprecipitation}

RiboTag IP or DAZL RIP analysis was performed as described previously ${ }^{18,}{ }^{19}$. Briefly, GVarrested or MI oocytes (200 oocytes/sample) were washed and collected in RNase-free PBS with $1 \%$ polyvinylpyrrolidone. After lysis in $300 \mu \mathrm{l}$ of supplemented homogenization buffer S-HB; 50 $\mathrm{mM}$ Tris- $\mathrm{HCl} \mathrm{pH} 7.4,100 \mathrm{mM} \mathrm{KCl}, 12 \mathrm{mM} \mathrm{MgCl}$, 1\% NP-40, $1 \mathrm{mM}$ dithiothreitol, protease inhibitors, $40 \mathrm{U}$ RNAseOUT, $100 \mu \mathrm{g} / \mathrm{ml}$ cycloheximide and $1 \mathrm{mg} / \mathrm{ml}$ heparin (Sigma-Aldrich, H3393)]., samples were centrifuged at $12,000 \mathrm{~g}$ for $10 \mathrm{mins}$ and supernatants were precleared with prewashed Protein G magnetic Dynabeads (Invitrogen, 10007D) for 30 min at $4{ }^{\circ} \mathrm{C} .15 \mu l$ of precleared lysates was aliquot for input (total transcripts) and stored at $-80^{\circ} \mathrm{C}$ for $\mathrm{mRNA}$ extraction in next day. The remain precleared lysates were incubated with specific antibody (anti-HA antibody, anti-DAZL antibody) or its corresponding $\lg$ (mouse $\lg$, ab37355; rabbit $\lg$, ab37415; Abcam) $4 \mathrm{hrs}$ at $4^{\circ} \mathrm{C}$ on a rotor. Then pre-washed Protein $\mathrm{G}$ magnetic Dynabeads were added in the lysates for overnight incubation at $4^{\circ} \mathrm{C}$ on a rotator. The following day, bead pellets were washed three times in $500 \mu \mathrm{l}$ homogenization buffer $(\mathrm{HB})$ on a rotor at $4^{\circ} \mathrm{C}$ for $10 \mathrm{~min}$. Two more washes were performed with $1 \mathrm{M}$ urea/high-salt buffer for $10 \mathrm{~min}$ each. RNA eluted from beads was either HA-tagged ribosome associated transcripts or IgG (no-specific binding 
524 transcripts), together with input for extraction. In some experiments, the specificity of the

525 immunoprecipitation was determined by using WT rather than RiboTag mice. RNA was purified

526 with RNeasy Plus Micro kit (Qiagen, 74034) according to manufacturer's instructions and directly

527 used for RNA-Seq analysis or reverse transcription for qPCR analysis. cDNA was prepared using

528 SuperScript III First-Strand Synthesis system (Invitrogen, 18080-051) with random hexamer

529 oligonucleotide primers. cDNA samples were stored $-80^{\circ} \mathrm{C}$ for following experiments.

530 For the RiboTag/qPCR analysis, Ccnb1, Dppa3 and Gdf9 (transcripts not regulated by DAZL as 531 previous reported ${ }^{18,19}$ ), were used to normalize the qPCR data. Zp3 contains no recognizable 532 DAZL-binding element and was used as negative controls for DAZL immunoprecipitation. The 533 data are reported as fold enrichment, with $\lg$ G values set to 1.

\section{Reporter mRNA preparation and reporter assay}

535 The Oosp1, Obox5, CenpE and Ccnb1 3'UTR sequences were obtained by sequencing oocyte 536 cDNA and cloned downstream of the YPet coding sequence. An oligo (A) stretch of $20 \mathrm{~A}$ was added in each construct. All constructs were prepared in the pcDNA 3.1 vector containing a T7 promoter, allowing for in vitro transcription to synthesize mRNAs, and fidelity was confirmed by DNA sequencing. mRNA reporters were transcribed in vitro to synthesize mRNAs with mMESSAGE mMACHINE T7 Transcription Kit (Ambion, AM1344); polyadenylation of mCherry was obtained using Poly(A) Tailing Kit (Ambion, AM1350). All the messages were purified using MEGAclear Kit (Ambion, AM1908). mRNA concentrations were measured by NanoDrop and its integrity was evaluated by electrophoresis.

544 Time-lapse recordings were performed using a Nikon Eclipse T2000-E equipped with mobile 545 stage and environmental chamber of $37^{\circ} \mathrm{C}$ and $5 \% \mathrm{CO}_{2}$. YFP-Oosp1, YFP-Obox5, YFP-Cenpe 546 or YFP-CcnB1 were injected at $12.5 \mathrm{ng} / \mu \mathrm{L}$ with either CON-MO or DAZL-MO. Each YFP-3'UTR reporters we also co-injected with polyadenylated mCherry at $12.5 \mu \mathrm{g} / \mu \mathrm{L}$ in oocyte. After injection, oocytes were pre-incubated overnight in $\alpha$-MEM medium supplemented with $2 \mu \mathrm{M}$ cilostamide to allow expression of the reporters. mCherry signals did not change significantly in oocytes at different stages of maturation. Ratios of YFP reporter and the level of mCherry signal measured at plateau in each oocyte were calculated. In those cases where DAZL ablation had an effect in 552 GV oocytes, the data were normalized to the signal of GV stage accumulation of corresponding 553 proteins. Rate of translation associate with reentering into cell cycle (after GVBD versus before 554 GVBD) were calculated by fitting YFP:mCherry data and calculating the slope of the interpolation 
555

556

557

558

559

560

561

562

563

564

565

566

567

568

569

570

571

572

573

574

575

576

577

578

579

580

581

582

583

584

obtained by linear regression (Prism) prior to GVBD or after GVBD when a new rate of translation had stabilized.

\section{DAZL RIP-Chip analysis}

DAZL RIP-Chip was performed as previously reported ${ }^{18}$. Briefly, C57BL/6 female mice (22-24 days old) were primed with PMSG, after $48 \mathrm{hrs,} \mathrm{mice} \mathrm{were} \mathrm{stimulated} \mathrm{with} \mathrm{hCG} \mathrm{for} 0 \mathrm{hr}, 6 \mathrm{hrs}$, or $14 \mathrm{hrs}$ to collect GV, MI, and MII stage oocytes. Oocyte lysates were centrifuged at 12,000 $\mathrm{g}$ for 10 mins at $4^{\circ} \mathrm{C}$. Supernatants were used for RNA extraction. RNA was purified with RNeasy Plus Micro kit (Qiagen). RNAs in the RNP fractions were reverse-transcribed with SuperScript III (Invitrogen). Five micrograms of cDNA was fragmented and hybridized with Affymetrix Mouse Genome 430.2 array chips ${ }^{46}$. DNA-Chip Analyzer (dChip) was used for normalization and to quantify microarray signals with default analysis parameters. Comparison between samples was performed using dChip with a fold change of 1.5 , FDR $<5 \%$, and $\mathrm{P}<0.05$.

\section{Statistical analysis}

Statistical analysis was performed using the GraphPad Prism8 package. The statistical analysis performed depended on specific experiment and is reported in the figure legend. For comparison between two groups, two-tailed paired t-test was used. Statistical significances is denoted by asterisk in each graph. The quality check of RNA-Seq reads was performed using FastQC and reads were then trimmed with Trimmomatic. Alignment of the reads to the mouse genome was performed by Hisat2, .bam files were sorted and indexed using Samtools, and count files were generated by HTSeq. TMM normalization and the remaining RNA-Seq statistical analyses were done through edgeR and other Bioconductor scripts.

\section{Acknowledgements}

We wish to thank Dr. Matthew Cook for the help in the RIP-Chip experiments. We thank Dr. Soeren Muller and Dr. Xiaoyuan Zhou for their help and advice on processing and analyzing the RNA-Seq data. This work was supported by NIH R01 GM097165, GM116926 and the Specialized Cooperative Centres Programme in Reproduction and Infertility Research (P50HD055764-10), Eunice Kennedy Shriver National Institute of Child Health and Human Development (NICHD) to MC. Enrico Maria Daldello is supported by a Fellowship from the Lalor Foundation. Xuan G. Luong is supported by a T32-HD007263 Integrated Training in Reproductive Sciences. 
588

589

590

591

592

593

594

595

596

597

598

599

600

601

602

603

604

605

606

607

608

609

610

611

612

613

614

615

616

617

618

619

620

621

622

623

624

625

626

627

628

629

630

631

632

633

634

\section{References}

1. Seydoux G, Braun RE. Pathway to totipotency: lessons from germ cells. Cell 127, 891904 (2006).

2. Schultz RM. From egg to embryo: a peripatetic journey Reproduction 130, 825-828 (2005).

3. Vardy L, Orr-Weaver TL. Regulating translation of maternal messages: multiple repression mechanisms. Trends Cell Biol 17, 547-554 (2007).

4. Sugimori S, Kumata Y, Kobayashi S. Maternal Nanos-Dependent RNA Stabilization in the Primordial Germ Cells of Drosophila Embryos. Dev Growth Differ 60, 63-75 (2018).

5. Clarke HJ. Post-transcriptional control of gene expression during mouse oogenesis. Results Probl Cell Differ 55, 1-21 (2012).

6. Kuersten S, Goodwin EB. The power of the 3' UTR: translational control and development. Nat Rev Genet 4, 626-637 (2003).

7. Weill L, Belloc E, Bava FA, Mendez R. Translational control by changes in poly(A) tail length: recycling mRNAs. Nat Struct Mol Biol 19, 577-585 (2012).

8. Radford HE, Meijer HA, de Moor $\mathrm{CH}$. Translational control by cytoplasmic polyadenylation in Xenopus oocytes. Biochim Biophys Acta 1779, 217-229 (2008).

9. Richter JD. CPEB: a life in translation. Trends Biochem Sci 32, 279-285 (2007).

10. Reynolds N, Cooke HJ. Role of the DAZ genes in male fertility. Reprod Biomed Online 10, $72-80$ (2005).

11. Brook M, Smith JW, Gray NK. The DAZL and PABP families: RNA-binding proteins with interrelated roles in translational control in oocytes. Reproduction 137, 595-617 (2009).

12. Lin Y, Page DC. Dazl deficiency leads to embryonic arrest of germ cell development in XY C57BL/6 mice. Developmental biology 288, 309-316 (2005).

13. Reynolds N, et al. Dazl binds in vivo to specific transcripts and can regulate the premeiotic translation of Mvh in germ cells. Hum Mol Genet 14, 3899-3909 (2005).

14. Collier B, Gorgoni B, Loveridge C, Cooke HJ, Gray NK. The DAZL family proteins are PABP-binding proteins that regulate translation in germ cells. The EMBO journal 24, 2656-2666 (2005).

15. Chen $\mathrm{HH}$, et al. DAZL limits pluripotency, differentiation, and apoptosis in developing primordial germ cells. Stem Cell Reports 3, 892-904 (2014).

16. Kobayashi M, Tani-Matsuhana S, Ohkawa $\mathrm{Y}$, Sakamoto H, Inoue K. DND protein functions as a translation repressor during zebrafish embryogenesis. Biochem Biophys Res Commun 484, 235-240 (2017). 
672

673

674

675

676

677

678

679

680

681

682

683

684

685

17. Zagore LL, et al. DAZL Regulates Germ Cell Survival through a Network of PolyAProximal mRNA Interactions. Cell Rep 25, 1225-1240 e1226 (2018).

18. Chen J, et al. Genome-wide analysis of translation reveals a critical role for deleted in azoospermia-like (Dazl) at the oocyte-to-zygote transition. Genes \& development 25, 755-766 (2011).

19. Sousa Martins JP, et al. DAZL and CPEB1 regulate mRNA translation synergistically during oocyte maturation. J Cell Sci 129, 1271-1282 (2016).

20. Fukuda K, Masuda A, Naka T, Suzuki A, Kato Y, Saga Y. Requirement of the 3'-UTRdependent suppression of DAZL in oocytes for pre-implantation mouse development. PLoS Genet 14, e1007436 (2018).

21. McNeilly JR, Watson EA, White YA, Murray AA, Spears N, McNeilly AS. Decreased oocyte DAZL expression in mice results in increased litter size by modulating folliclestimulating hormone-induced follicular growth. Biology of reproduction 85, 584-593 (2011).

22. Welling $M$, et al. DAZL regulates Tet1 translation in murine embryonic stem cells. EMBO Rep 16, 791-802 (2015).

23. Percharde M, Bulut-Karslioglu A, Ramalho-Santos M. Hypertranscription in Development, Stem Cells, and Regeneration. Dev Cell 40, 9-21 (2017).

24. Yan C, Pendola FL, Jacob R, Lau AL, Eppig JJ, Matzuk MM. Oosp1 encodes a novel mouse oocyte-secreted protein. Genesis 31, 105-110 (2001).

25. Rajkovic A, Yan C, Yan W, Klysik M, Matzuk MM. Obox, a family of homeobox genes preferentially expressed in germ cells. Genomics 79, 711-717 (2002).

26. Jenkins HT, Malkova B, Edwards TA. Kinked beta-strands mediate high-affinity recognition of mRNA targets by the germ-cell regulator DAZL. Proc Natl Acad Sci U S A 108, 18266-18271 (2011).

27. Zeng M, et al. DAZL binds to 3'UTR of Tex19.1 mRNAs and regulates Tex19.1 expression. Mol Biol Rep 36, 2399-2403 (2009).

28. Ollinger $\mathrm{R}$, et al. Deletion of the pluripotency-associated Tex19.1 gene causes activation of endogenous retroviruses and defective spermatogenesis in mice. PLoS Genet 4, e1000199 (2008).

29. MacLennan M, et al. Mobilization of LINE-1 retrotransposons is restricted by Tex19.1 in mouse embryonic stem cells. Elife 6, (2017).

30. Tarabay $\mathrm{Y}$, et al. The mammalian-specific Tex19.1 gene plays an essential role in spermatogenesis and placenta-supported development. Hum Reprod 28, 2201-2214 (2013).

31. Yu C, et al. BTG4 is a meiotic cell cycle-coupled maternal-zygotic-transition licensing factor in oocytes. Nat Struct Mol Biol 23, 387-394 (2016). 
686

687

688

689

690

691

692

693

694

695

696

697

698

699

700

701

702

703

704

705

706

707

708

709

710

711

712

713

714

715

716

717

718

719

720

721

722

723

724

725

726

727

728

729

730

731

732

733

734
32. Pasternak M, Pfender S, Santhanam B, Schuh M. The BTG4 and CAF1 complex prevents the spontaneous activation of eggs by deadenylating maternal mRNAs. Open biology 6, (2016).

33. Liu Y, et al. BTG4 is a key regulator for maternal mRNA clearance during mouse early embryogenesis. J Mol Cell Biol 8, 366-368 (2016).

34. Wu D, Dean J. BTG4, a maternal mRNA cleaner. J Mol Cell Biol 8, 369-370 (2016).

35. Rosario R, Smith RW, Adams IR, Anderson RA. RNA immunoprecipitation identifies novel targets of DAZL in human foetal ovary. Mol Hum Reprod 23, 177-186 (2017).

36. Wiszniak SE, Dredge BK, Jensen KB. HuB (elavl2) mRNA is restricted to the germ cells by post-transcriptional mechanisms including stabilisation of the message by DAZL. PLoS One 6, e20773 (2011).

37. Cheng MH, Maines JZ, Wasserman SA. Biphasic subcellular localization of the DAZLrelated protein boule in Drosophila spermatogenesis. Dev Biol 204, 567-576 (1998).

38. Sha $Q Q$, et al. A MAPK cascade couples maternal mRNA translation and degradation to meiotic cell cycle progression in mouse oocytes. Development 144, 452-463 (2017).

39. Robu ME, et al. p53 activation by knockdown technologies. PLoS Genet 3, e78 (2007).

40. Danilova N, Kumagai A, Lin J. p53 upregulation is a frequent response to deficiency of cell-essential genes. PLoS One 5, e15938 (2010).

41. Moulton JD. Making a Morpholino Experiment Work: Controls, Favoring Specificity, Improving Efficacy, Storage, and Dose. Methods Mol Biol 1565, 17-29 (2017).

42. Rossi A, et al. Genetic compensation induced by deleterious mutations but not gene knockdowns. Nature 524, 230-233 (2015).

43. Padmanabhan K, Richter JD. Regulated Pumilio-2 binding controls RINGO/Spy mRNA translation and CPEB activation. Genes Dev 20, 199-209 (2006).

44. Mendez R, Hake LE, Andresson T, Littlepage LE, Ruderman JV, Richter JD.

Phosphorylation of CPE binding factor by Eg2 regulates translation of c-mos mRNA. Nature 404, 302-307 (2000).

45. Tung JY, et al. Novel missense mutations of the Deleted-in-AZoospermia-Like (DAZL) gene in infertile women and men. Reprod Biol Endocrinol 4, 40 (2006).

46. Su YQ, et al. Selective degradation of transcripts during meiotic maturation of mouse oocytes. Dev Biol 302, 104-117 (2007). 


\section{Figure Legends}

736

737

738

739

740

741

742

743

744

745

746

747

748

749

750

751

752

753

754

755

756

757

758

759

760

761

762

763

764

765

Figure 1. Interference with DazI mRNA translation depletes the oocyte of the DAZL protein and inhibits translation of a specific downstream target

(a) DAZL protein accumulation during the transition from the GV-to-MI stage of oocyte maturation. Accumulation of $\alpha$-tubulin was used as a loading control. GV stage oocytes from wild type mice were cultured in vitro for maturation. After 0-8 hrs maturation, oocytes were collected, lysed in sample buffer, and used for Western blot analysis. 150 oocytes per lane was loaded in this experiment. (b) Morpholino down-regulation of DAZL protein. GV stage oocytes from wild type or DAZL Heterozygous mice were injected with CON or DAZL MO. Oocytes were preincubated overnight with $2 \mu \mathrm{M}$ milrinone and then cultured in inhibitor-free medium for maturation. After 6hrs, oocytes were collected and used for Western blot analysis. A representative experiment of the three performed is reported. (c-e) Ribosome loading of endogenous Dazl and Tex19.1, but not CcnB1, mRNA is blocked after DAZL depletion. GV stage oocytes from wild type or Dazl+/- mice were injected with CON-MO or Dazl MO and preincubated overnight in $2 \mu \mathrm{M}$ milrinone, then cultured in inhibitor-free medium for maturation. Oocytes were collected at $0 \mathrm{hr}$ and $6 \mathrm{hrs}$ for RiboTag IP followed by qPCR analysis. (GV:germinal vesicle; MI:Meiosis I). Each dot represents the average of triplicate measurements from independent biological samples collected in different days. ** $\mathrm{P}<0.01 ;{ }^{* \star * *} \mathrm{P}<0.0001$.

\section{Figure 2. Maternal mRNA loading onto ribosome is disrupted in oocytes depleted of DAZL}

(a) Comparison of the transcriptomes of oocytes injected with control and Daz/ MO. Oocytes from wild type mice were injected with a CON-MO whereas oocytes from heterozygous Dazl mice were injected with a DAZL-MO. Oocytes were incubated overnight in the presence of milrinone and the following morning were collected for RiboTag IP/RNASeq as described in the 'Materials and Methods'. The average input (total transcripts) CPM data from duplicate biological replicates is reporterd. (b) Comparison of transcripts recovered by RiboTag IP/RNASeq in Control and DazI MO injected oocytes (MI). GV stage oocytes from wild type or $\mathrm{Daz}^{+/-}$mice were injected with CON-MO or Dazl-MO. After overnight preincubation with $2 \mu \mathrm{M}$ milrinone, oocytes were cultured in inhibitor-free medium to allow reentry into the meiotic cell cycle. Oocytes were collected at $6 \mathrm{hrs}$ for RiboTag IP and RNA-Seq analysis as detailed in the methods. Ribosome loading of the majority of transcripts present in the oocyte is not significantly changed after DAZL removal (grey dots). Ribosome loading of a subgroup (551 transcripts) of mRNAs (blue, FDR < 0.05 ) is 
766

767

768

769

770

771

772

773

774

775

776

777

778

779

780

781

782

783

784

785

786

787

788

789

790

791

792

793

794

795

796

797

significantly decreased, while ribosome loading of a distinct subgroup (170) of transcripts is significantly increased after DAZL removal (red dots, FDR < 0.05). (c-e) effect of DAZL depletion on RNA levels and ribosome loading of representative DAZL interacting targets. (c) DAZL RIPChip of oocyte extracts immunoprecipitation of selected mRNAs is reported as the fold enrichment DAZL AB/lgG N=3. (d and e) GV stage oocytes from wild type or Dazl+/- mice were injected with control or Dazl MOs. After overnight preincubation with $2 \mu \mathrm{M}$ milrinone, oocytes were cultured in inhibitor-free medium for maturation. Oocytes were collected at $0 \mathrm{hr}(\mathrm{GV})$ and $6 \mathrm{hrs}(\mathrm{MI})$ for RiboTag IP and RNA-Seq analysis. (d) RNASeq data from supernatants (input) from RiboTag IP of control and DazlMO (e) RiboTag IP/RNASeq analysis documented an increase in ribosome loading onto these transcripts (Tex19.1 and Txnip) in control oocytes but the increase is absent after DAZL KD. Akap10 and Nsf mRNA translation is increased after DAZL depletion.

Figure 3. RiboTag IP/qPCR confirms the presences of a subset of transcripts whose translation is upregulated and downregulated in oocytes depleted of DAZL.

Representative targets affected by DAZL removal in RiboTag/RNA-Seq dataset are showed in panel (a) (transcripts whose translation is downregulated by DAZL removal) and c (transcripts whose translation is upegulated by DAZL removal); the differences in ribosome loading DAZL $\mathrm{MO} / \mathrm{CON}-\mathrm{MO}$ for the same transcripts assessed in independent biological replicates by RiboTag IP/qPCR is reported in panel (b) and (d). Dppa3 and Ccnb1 are used as negative control in panel (a) and (b) for the transcripts whose translation is downregulated by DAZL removal, as they are not regulated by DAZL during oocyte maturation. Gdf9 mRNA is used as negative control in panel (c) and (d) for the transcripts whose translation is upregulated by DAZL removal, as it is not regulated by DAZL during oocyte maturation. Wild type and $\mathrm{Daz}^{+/-}$mice were injected with control or DAZL-MO. After overnight preincubation with $2 \mu \mathrm{M}$ milrinone, oocytes were cultured in inhibitorfree medium for maturation. Oocytes were collected at 6 hrs for RiboTag IP and qPCR analysis.

\section{Figure 4. DAZL interacts with transcripts whose translation is upregulated or} downregulated during oocyte maturation

a. Comparison DAZL TipChip/RiboTag IP RNseq in oocytes. Changes in ribosome loading from $0 \mathrm{hr}(\mathrm{GV})$ to $16 \mathrm{hrs}$ (MII stage) of DAZL targets assessed by RIP-Chip. A subset of transcripts whose translation increased from GV to MII stage are also enriched in DAZL immunoprecipitates of oocyte extracts (red); transcripts whose translation decreases during oocyte maturation are specifically immunoprecipiated by DAZL Antibody (blue). wild type oocytes were collected at $\mathrm{Oh}$ and 6hrs of in vitro maturation for RiboTag IP/RNASeq analysis. For DAZL RIP-Chip, wild type 
798

799

800

801

802

803

804

805

806

807

808

809

810

811

812

813

814

815

816

817

818

819

820

821

822

823

824

825

826

827

828

829

oocytes were primed with PMSG and after stimulation with hCG, MIl stage oocytes were harvested as described. Oocyte lysates was immunoprecipited with DAZL-specific antibody or $\lg G$ and the mRNA recovered in the IP pellet measured by microarray hybridization. (RIP-Chip data kindly provided by Jing Chen and Mat Cook members of the lab) (b) DAZL RNA-IP qPCR of ES cells extracts. ES cells were cultured in DMEM medium with supplements include $15 \%$ KOSR, 2\% FBS, 1x MEM Non-Essential Amino Acids (100x), 1x 2-mercaptoethanol, 10-7 U/ml

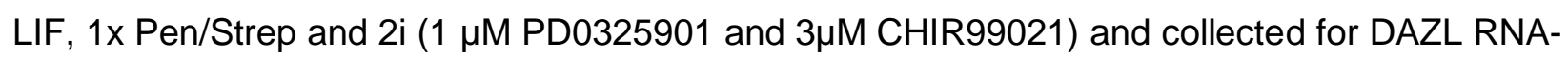
IP/qPCR analysis. The results are normalized with IgG IP. Asterisks $\left(^{*}\right)$ indicate that these transcripts are also found to be associated with DAZL also in the RIP-Chip dataset.

\section{Figure 5. The 3' UTR of Oosp1 and Obox5 recapitulates the effect of DAZL depletion on mRNA translation}

Oocytes were injected with 12.5ng/uL mCherry-polyadenylated mRNA and 12.5ng/uL YFPOosp1 3'UTR reporter for or YFP-Obox5 3'UTR reporter with either CON-MO or DAZL-MO. Oocytes were then pre-incubated overnight to allow the mCherry signal to reach a plateau. At the end of the preincubation, oocytes were released in cilostamide-free medium for maturation and YFP and mCherry signal were recorded by time lapse microscopy at a sampling rate of $30 \mathrm{~min}$ for 12 hrs. The YFP signal were corrected by the level of coinjected mCherry signal and normalized to the first reconrding of YFP/mCherry. Experiments were repeated three times and the data are the cumulative mean \pm SEM of three independent experiments. DAZL depletion decrease translation of reporter driven by the Oosp1 (a) or Obox5 (c) 3'UTR during oocyte maturation, while YFP-CcnB1 3'UTR (e) is not affected. Individual oocyte YFP/mCherry were used to calculate the rate of translation of the reporters at the 0-2 hrs (prior to GVBD) and 5-10 hrs (after GVBD). The rates of YFP-Oosp1 (b) ( $p<0.0001)$ or YFP-Obox5 (d) $(p<0.0001)$ translational accumulation are significantly decreased in GVBD after DAZL removal, whereas the rates of YFP-CcnB1 (f) translation are not significantly changed.

\section{Figure 6. The translation of the YFP-Oosp1 reporter is rescued by DAZL protein}

(a) Human DAZL protein injection restores Oosp1 translation during oocyte maturation in $\mathrm{MO}$ injected oocytes. Oocytes were injected with 12.5ng/uL mCherry mRNA and12.5ng/uL YFPOosp1 3' UTR reporter with either CON-MO or DAZL-MO with or without recombinant human DAZL protein, and incubated in cilostamide medium overnight to allow mCherry signal to reach a plateau. At the end of the preincubation, oocytes were released in cilostamide-free medium for maturation and YFP and mCherry signal recorded by time lapse microscopy at a sampling rate of 
30 mins for $12 \mathrm{hrs}$. YFP signal were corrected by the level of co-injected mCherry signal and were normalized to the first time point. Experiments were repeated 3 times and the data are the mean \pm SEM of three independent experiments. (b) Human DAZL protein injection restores the rate of YFP-Oosp1 translation with the effect of DAZL depletion in GVBD to levels of CON-MO. (c) Microinjection of a human DAZL protein rescues the meiotic block of oocytes injected with DAZLMO. Oocytes maturation was scored by counting the number of oocytes with a polar body. The injection of this human DAZL protein restored polar body formation to levels not significantly different from control (63\% versus 70\%). Three independent experiments were performed and the data reported here are the average ratio of polar body extrusion.

\section{Figure 7. DAZL depletion increases translation of Oosp1 and Obox5 endogenous} transcripts and Oosp1 and Obox5 reporters in GV-arrested oocytes.

(a, c, e) GV stage oocytes from wild type or Dazl+/- mice were injected with CON-MO or DAZL$\mathrm{MO}$. Oocytes were preincubated overnight with $2 \mu \mathrm{M}$ milrinone and then cultured in inhibitor-free medium for maturation. Ohr (GV stage) data from RiboTag IP/ RNA-Seq was used for Tra nslational efficiency (TE) analysis. TE was calculated as the ratio of the CPM for HA immunoprecipitated transcripts Oosp1 or Obox5 over the corresponding input at $0 \mathrm{hr}$ oocyte. The TEs for Oosp1 (a) and Obox5 (c) is increased in GV oocytes depleted of DAZL. However, TE for CcnB1 (e) is not affected. (b, d, f) GV stage oocytes were injected with 12.5ng/uL mCherrypolyadenylated mRNA and 12.5ng/uL YFP-3' UTR reporter for Oosp1 3' UTR or Obox5 3'UTR with either COMN-MO or DAZL-MO. Oocytes were pre-incubated overnight to allow mCherry signal to plateau, then released in cilostamide-free medium. YFP signal were corrected by the level of coinjected mCherry signal. The translation of both reporters in GV-arrested oocytes is significantly increased Oosp1 ( $p<0.0001)(b)$ and Obox5 ( $p=0.0007)(d)$ in DAZL-MO injection group, whereas no significant difference in the translation of the CcnB1 reporter (f) is detected. Experiments were repeated three times and the data reported are the rates for each individual oocytes from three independent biological replicates.

Figure 8. Translation of Oosp1 and Obox5 reporter is dependent on the presence of a DAZL binding element.

Constructs with the mutated DAZL-binding sequence, with wild type Oosp1 3' UTR, or with the Obox5 3'UTR along with mCherry-polyadenylated mRNA were injected into GV stage oocytes at $12.5 \mathrm{ng} / \mathrm{uL}$ per reporter. After overnight pre-incubation to allow mCherry signal to plateau, oocytes were released in cilostamide-free medium and recorded under the microscope for tracking YFP- 
862 Oosp1 or YFP-Obox5 translation during oocyte maturation. YFP and mCherry images were

863 acquired every 30 mins for $18 \mathrm{hrs}$. YFP signal were corrected by the level of co-injected mCherry

864 signal. Experiments were repeated three times on different days. (a) Scheme of the Oosp1 and

865 Obox5 3' UTR and position of the PAS, and putative CPEB and DAZL-binding elements.

866 Mutagenesis of the putative DAZL-binding element was performed as detailed in 'Materials and

867 Methods' sectioni. A red oval is the DAZL consensus sequence in the 3'UTR of Oosp1 and Obox5.

868 A black cross indicates the mutated DAZL-binding consensus sequence. (b and $\mathbf{e}$ ) The effect of

869 DAZL-binding element mutation on Oosp1 $(p=0.0062)$ or Obox5 $(p<0.0001)$ translation in GV

870 stage. Rates of reporter accumulation were calculated in each GV-arrested oocyte and plotted as

871 individual dots. Mean and SEM values were calculated from all the oocytes measured from three

872 different experiments. (c and f) Mutation of DAZL-binding element on 3'UTR of Oosp1 or Obox5

873 decreases translation of each respective reporter during oocyte maturation. YFP signals were

874 corrected by the level of co-injected mCherry signal. Every time point was normalized to the first

875 time point of YFP:mCherry. (d and $\mathbf{g})$ The rates of YFP-Oosp1 $(p<0.0001)$ or YFP-Obox5 $(p<$

876 0.0001) reporter accumulation during maturation is significantly decreased in GVBD after

877 mutation of the putative DAZL site.

878

879 
a

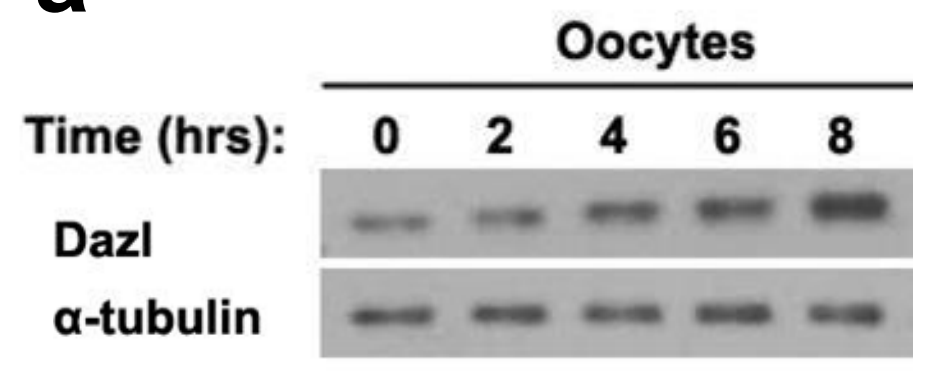

C

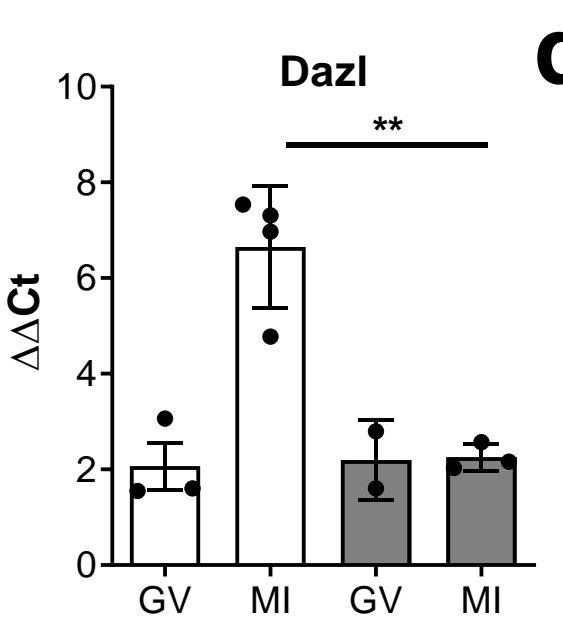

b
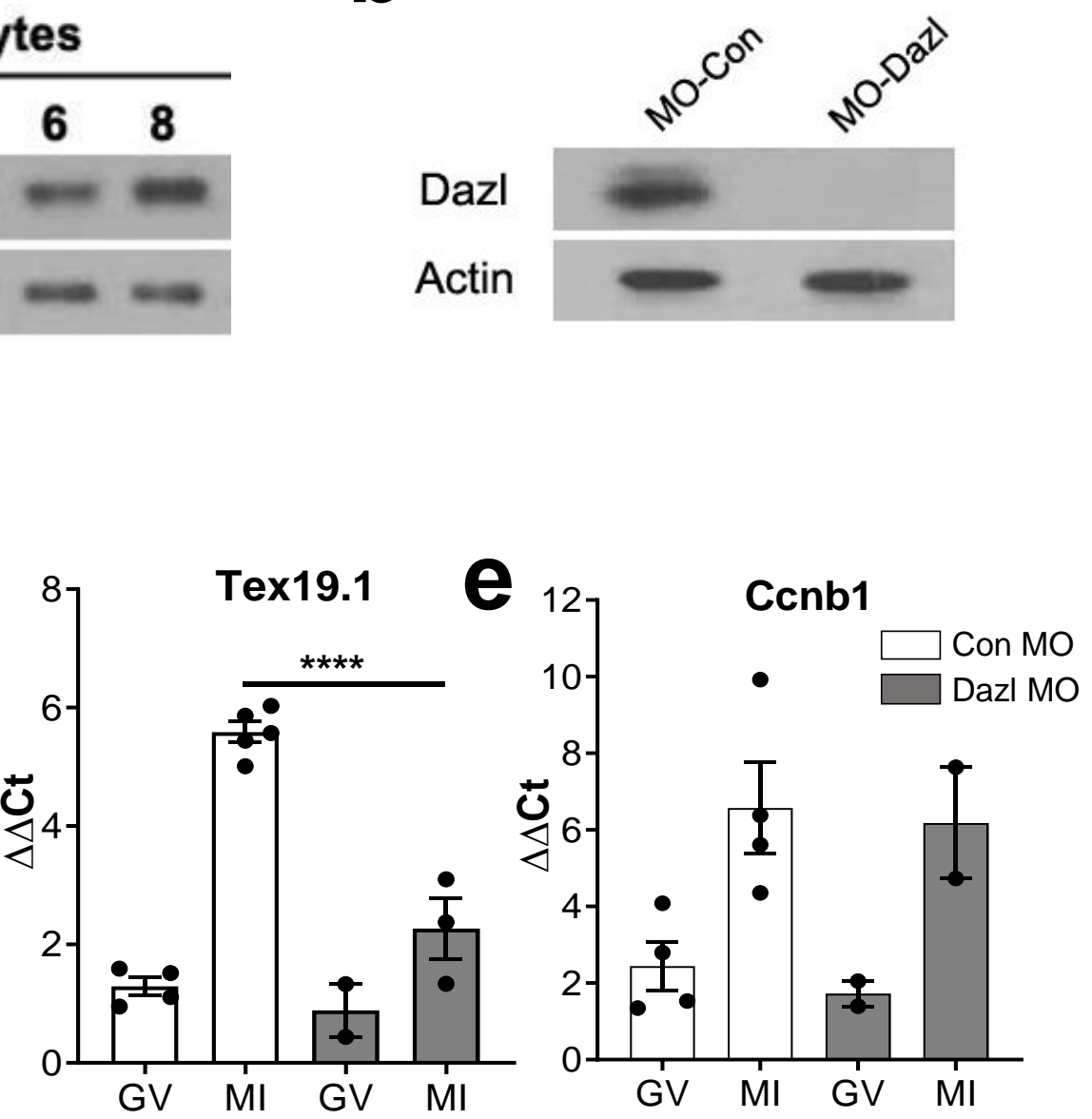
a
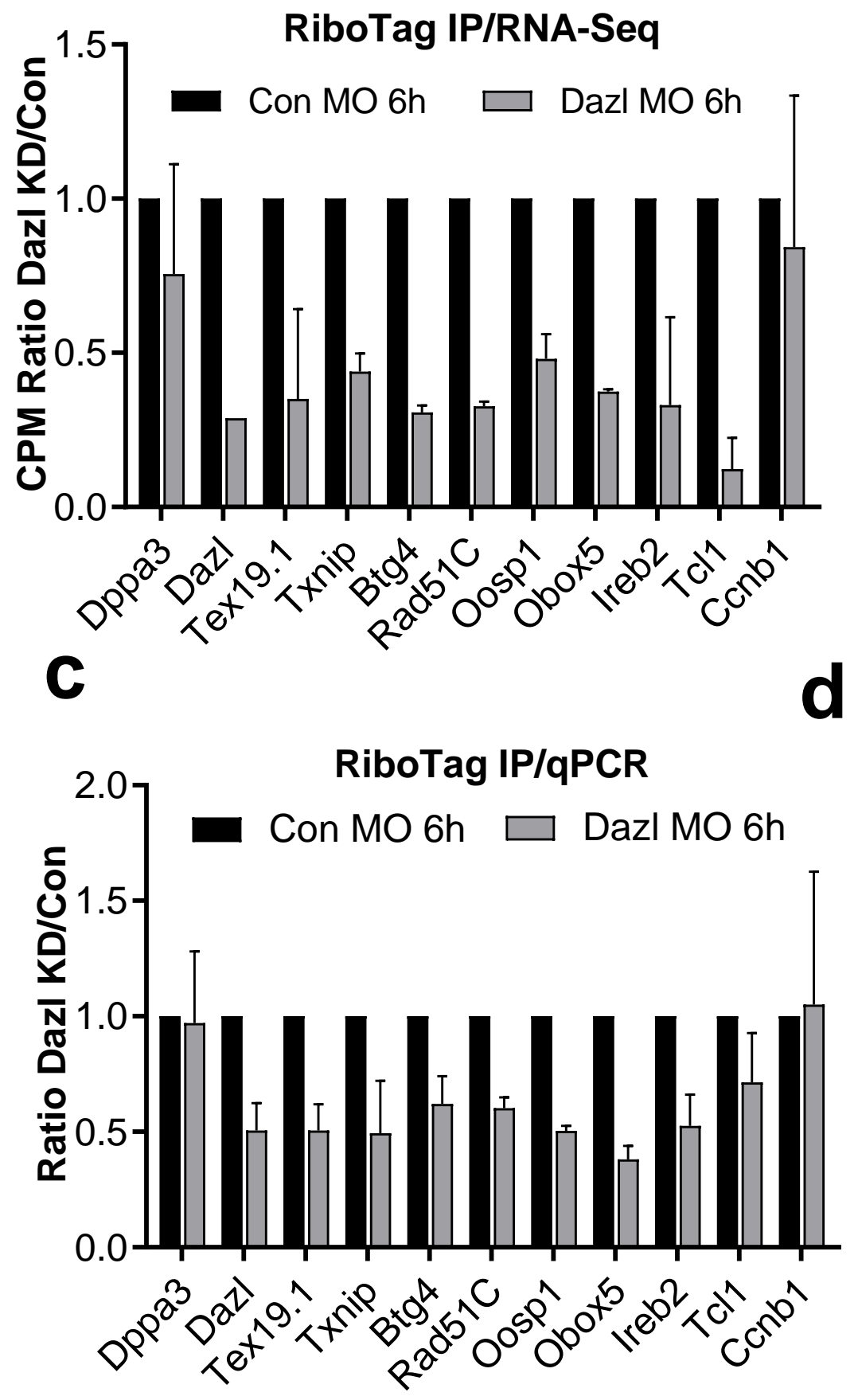

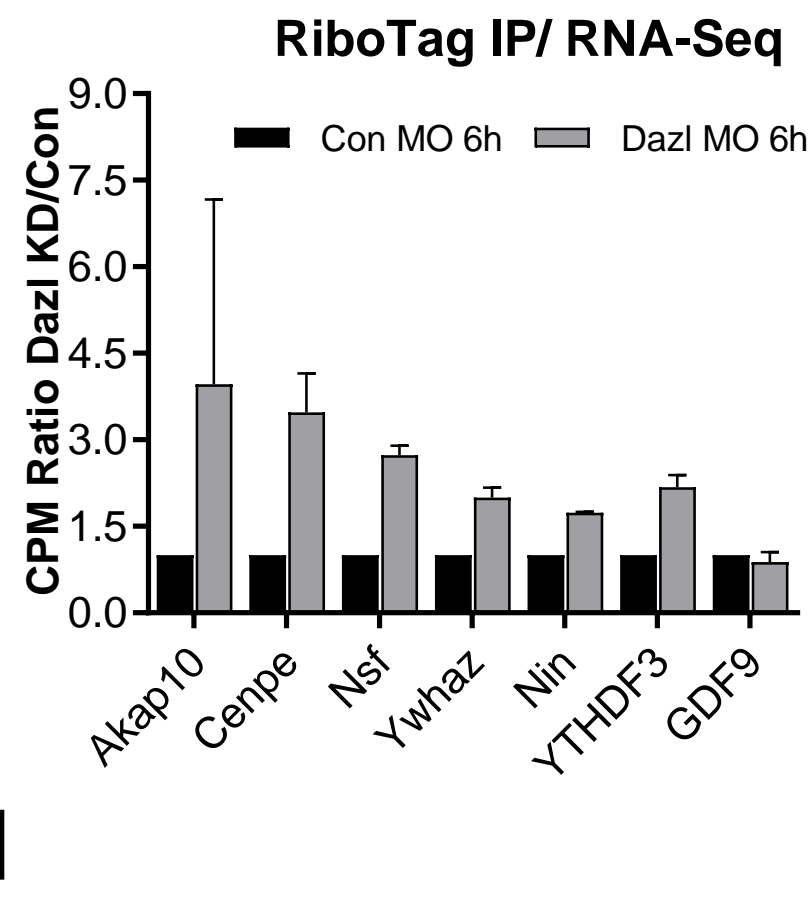

RiboTag IP/qPCR

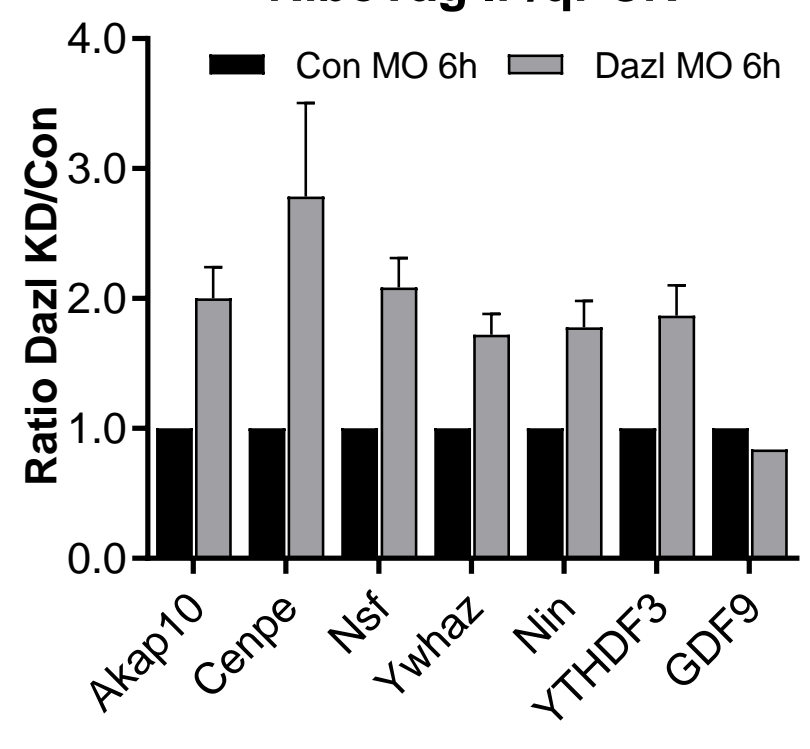


a

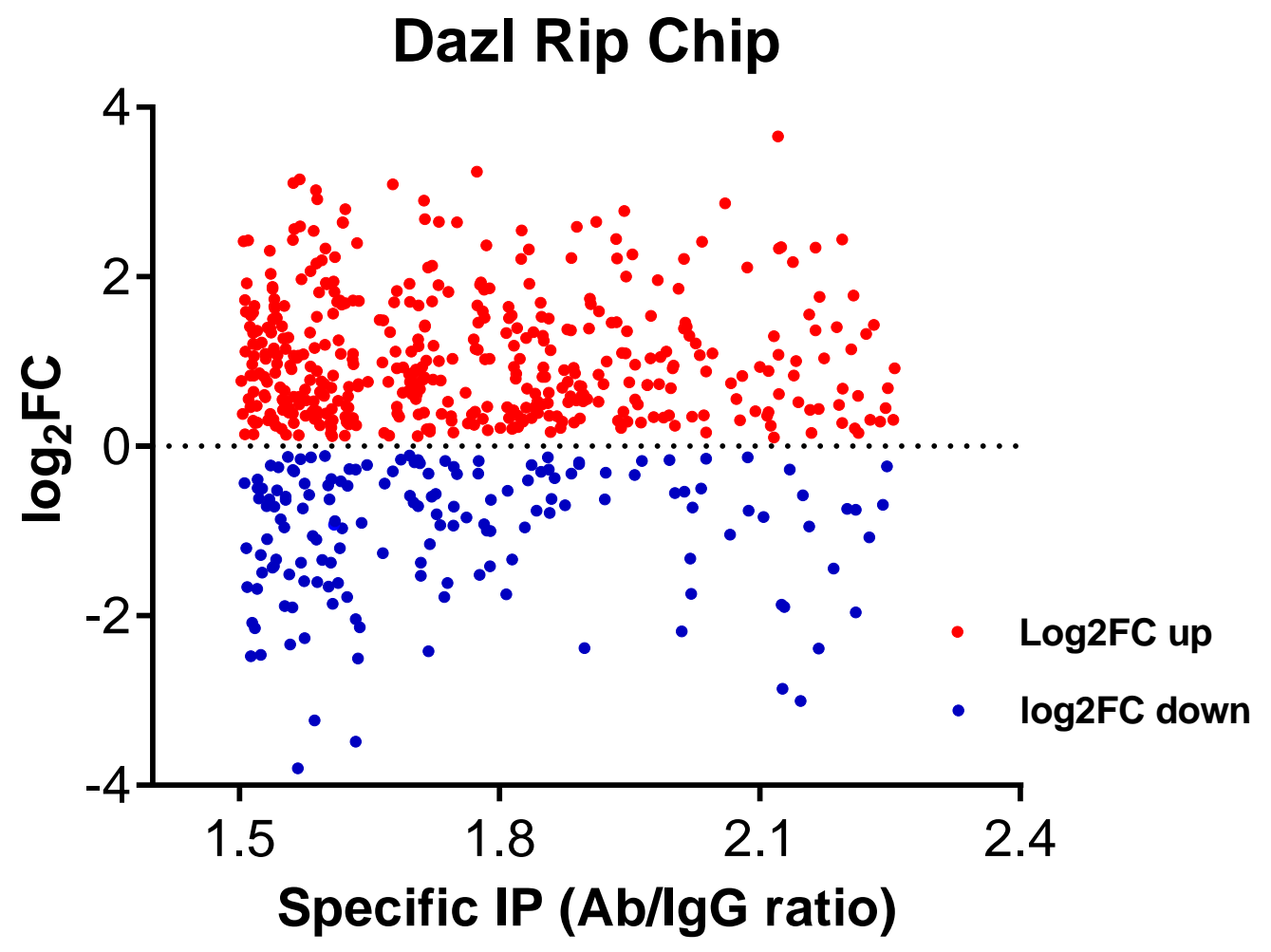

b

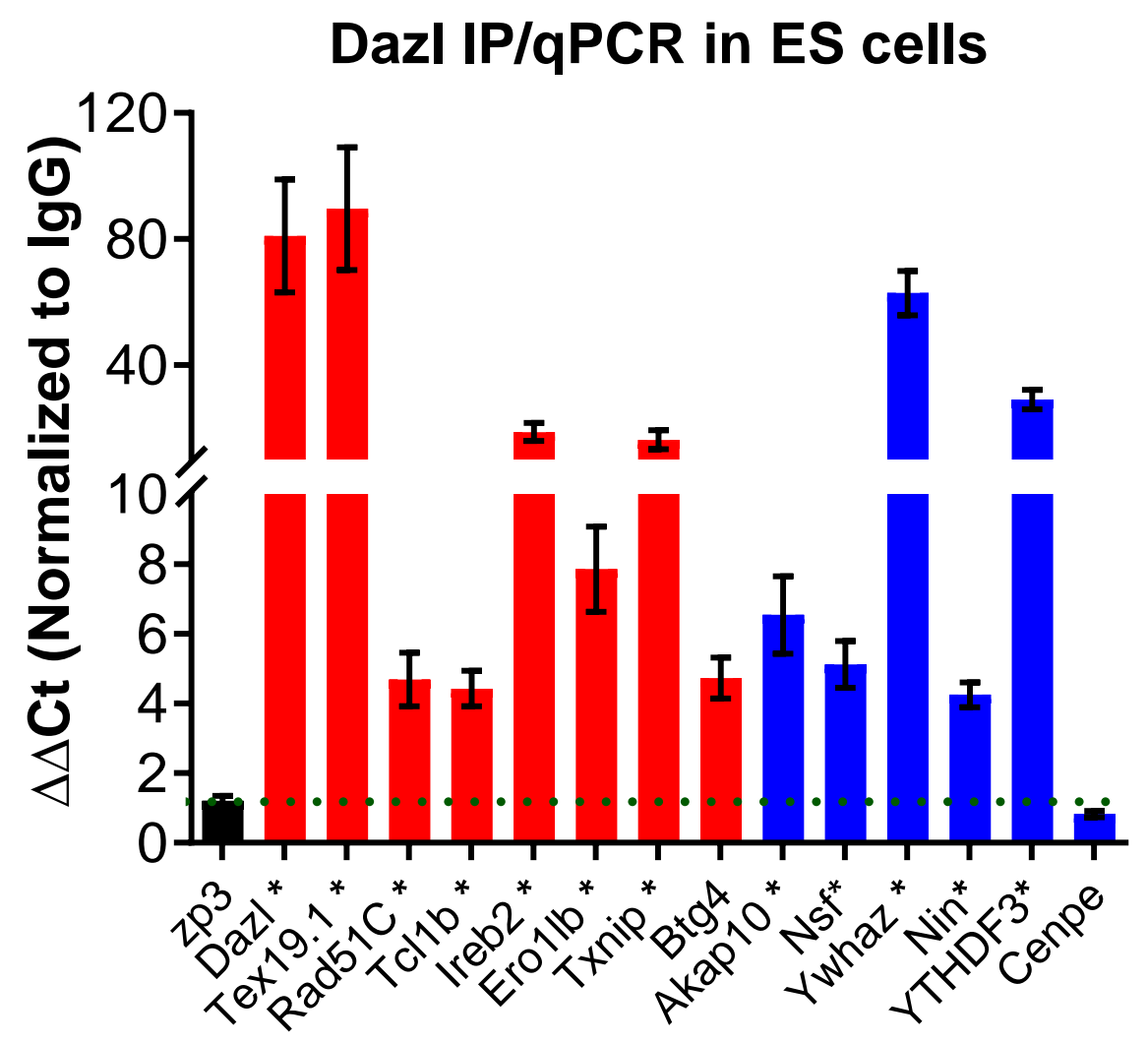




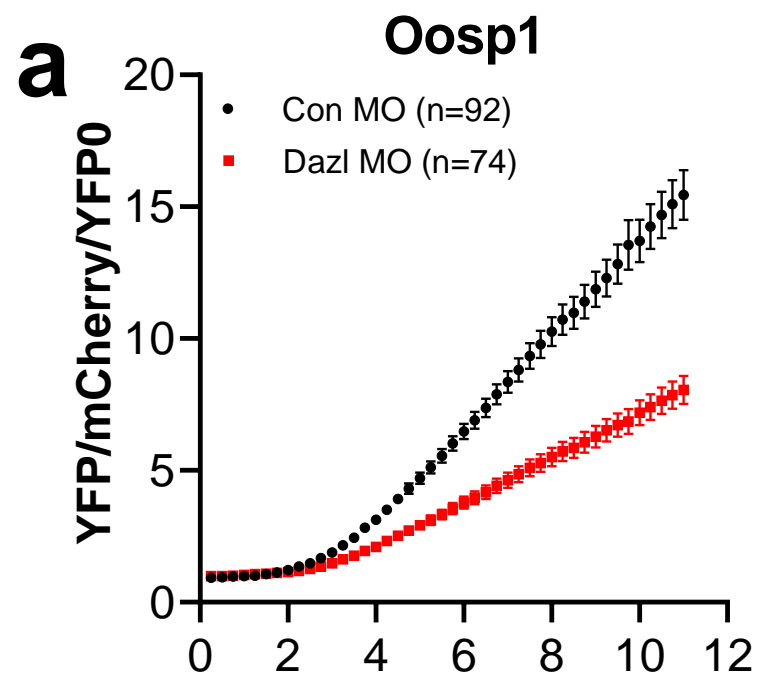

Time After Cilostamide Release (hrs)

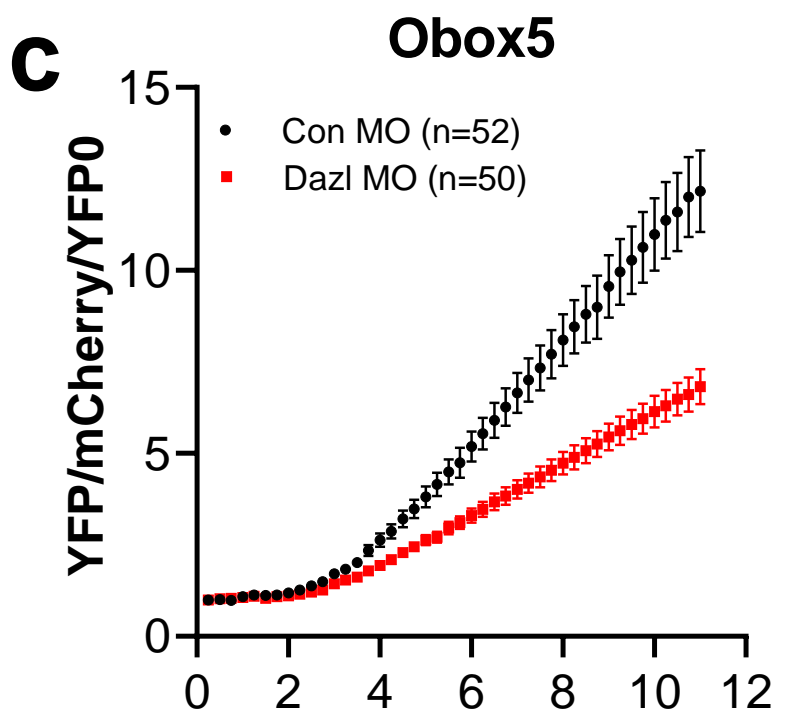

Time After Cilostamide Release (hrs)

e

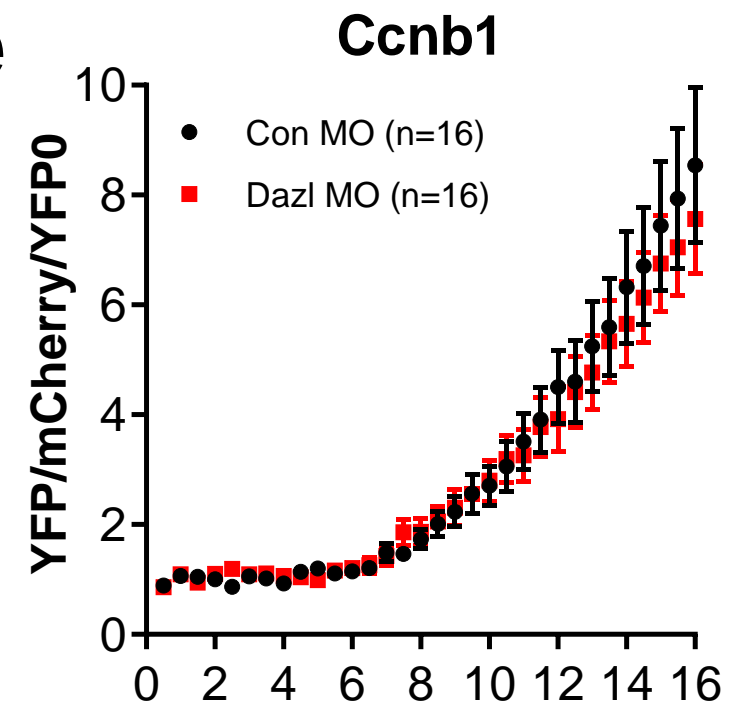

Time After Cilostamide Release (hrs)
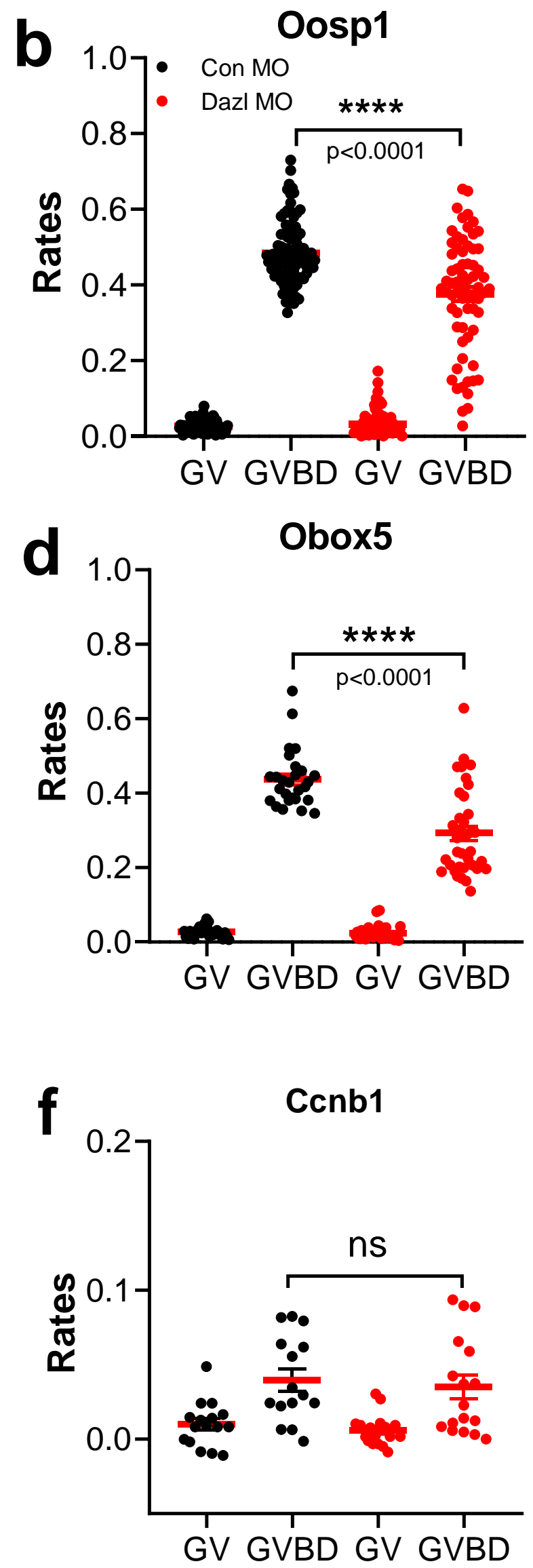
a

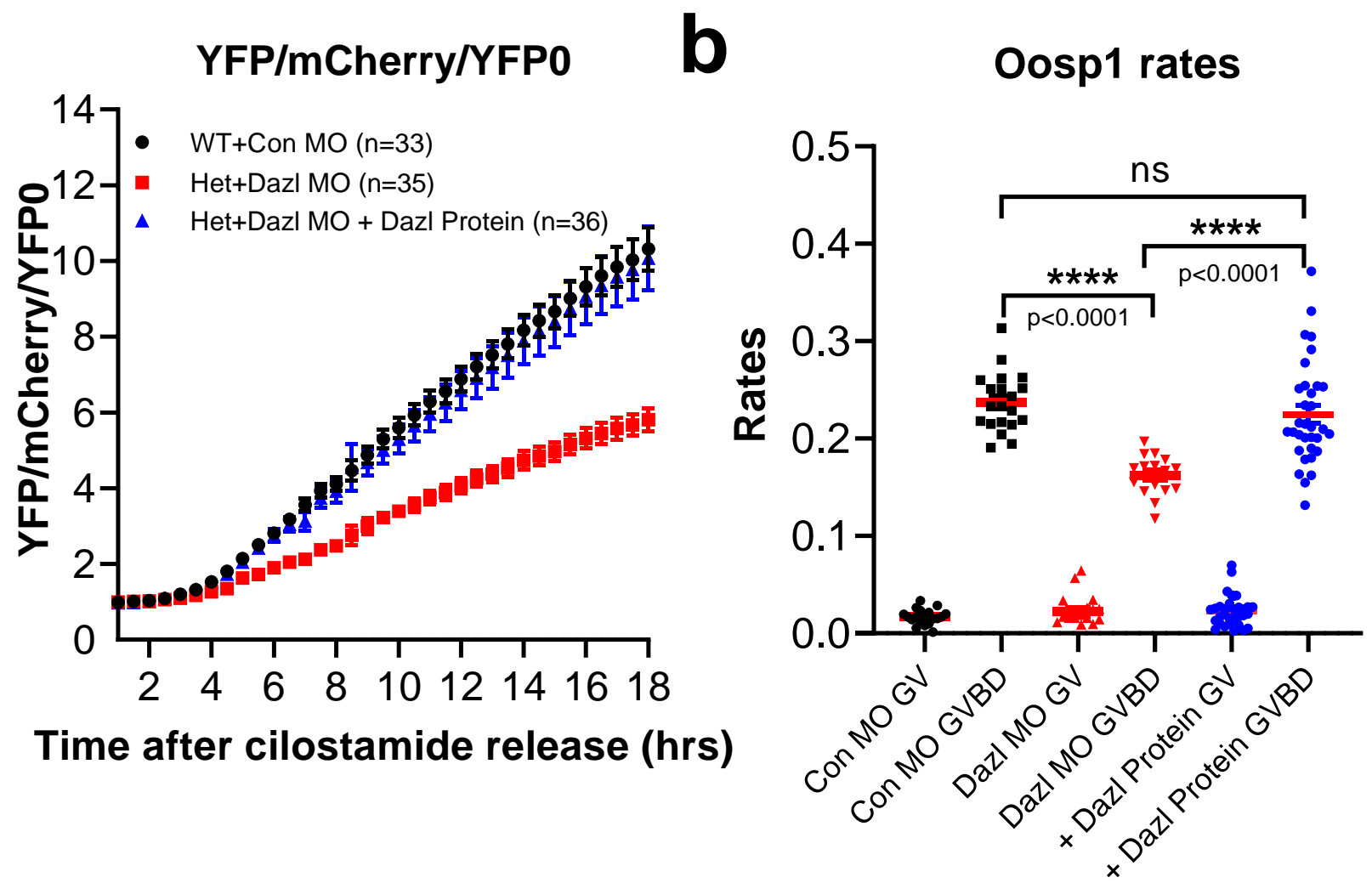

C

Polar body extrusion

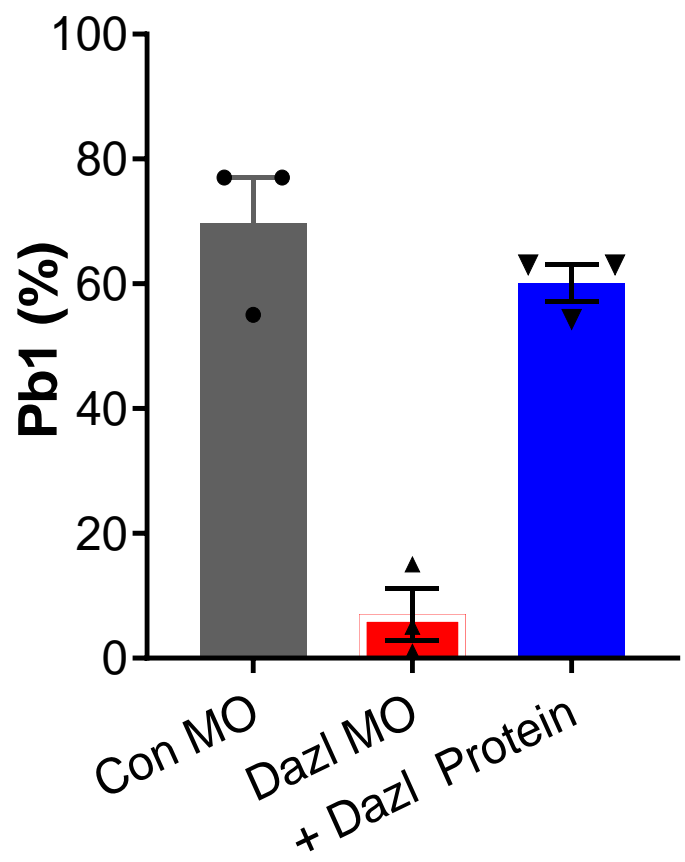



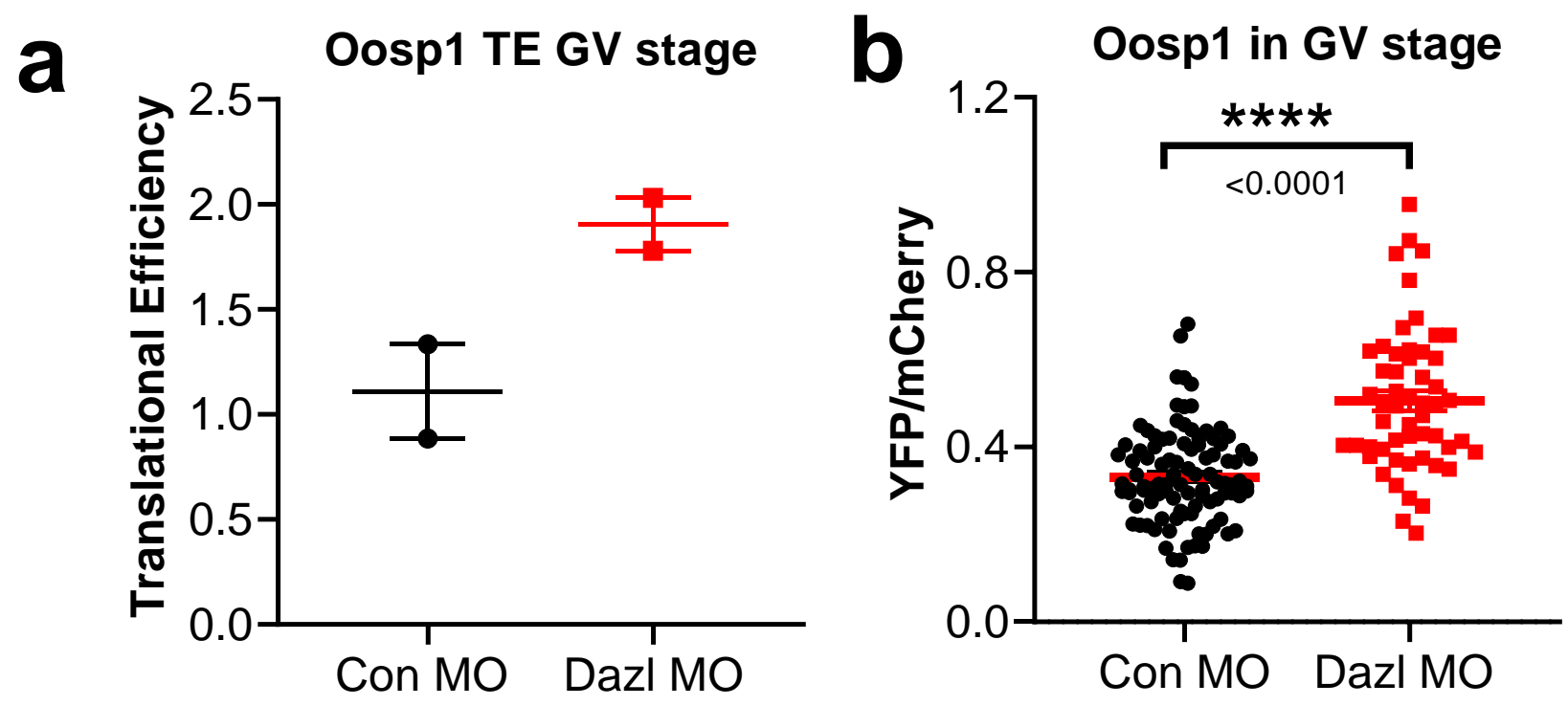

C

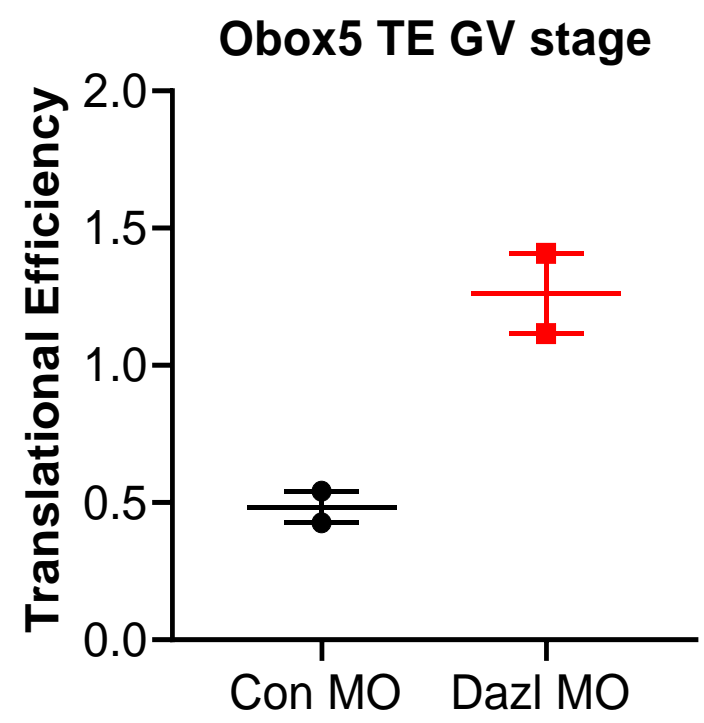

d

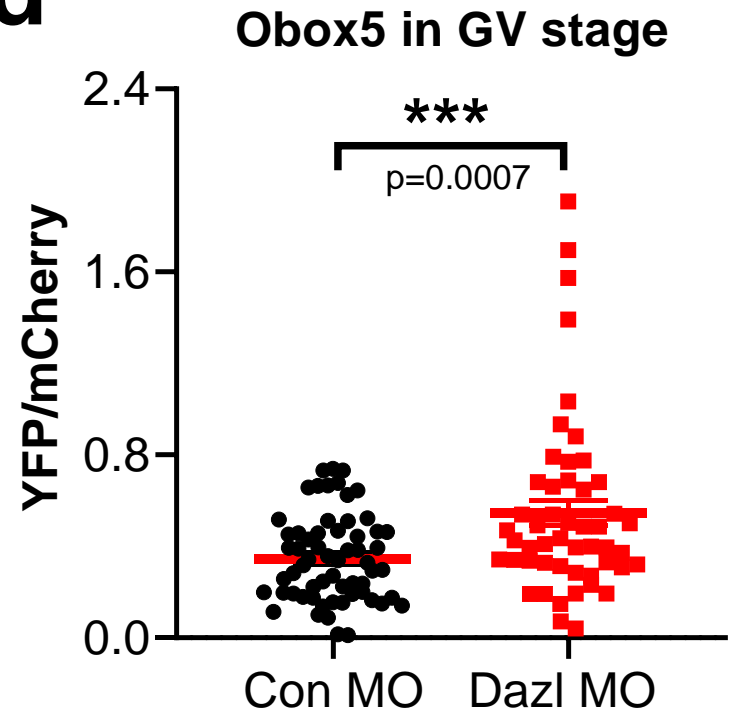

Ccnb1 TE GV stage
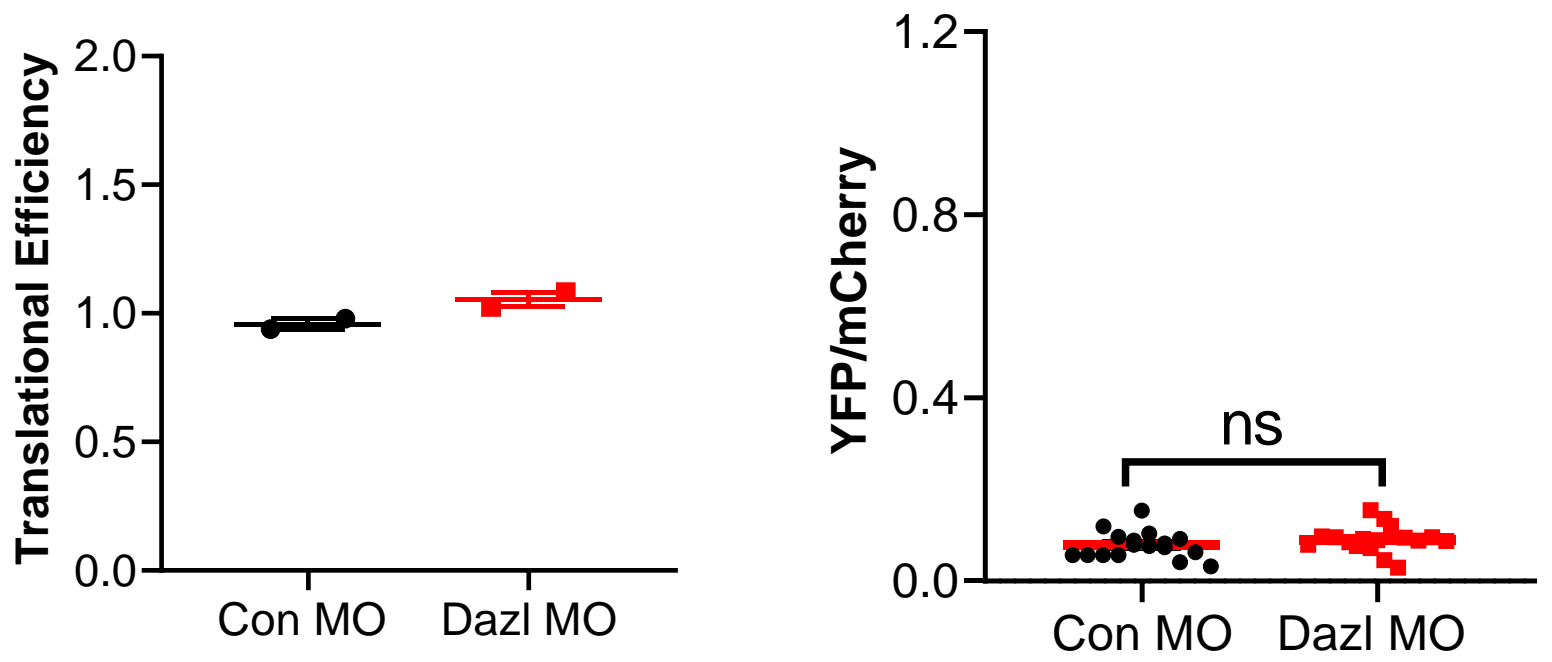
a

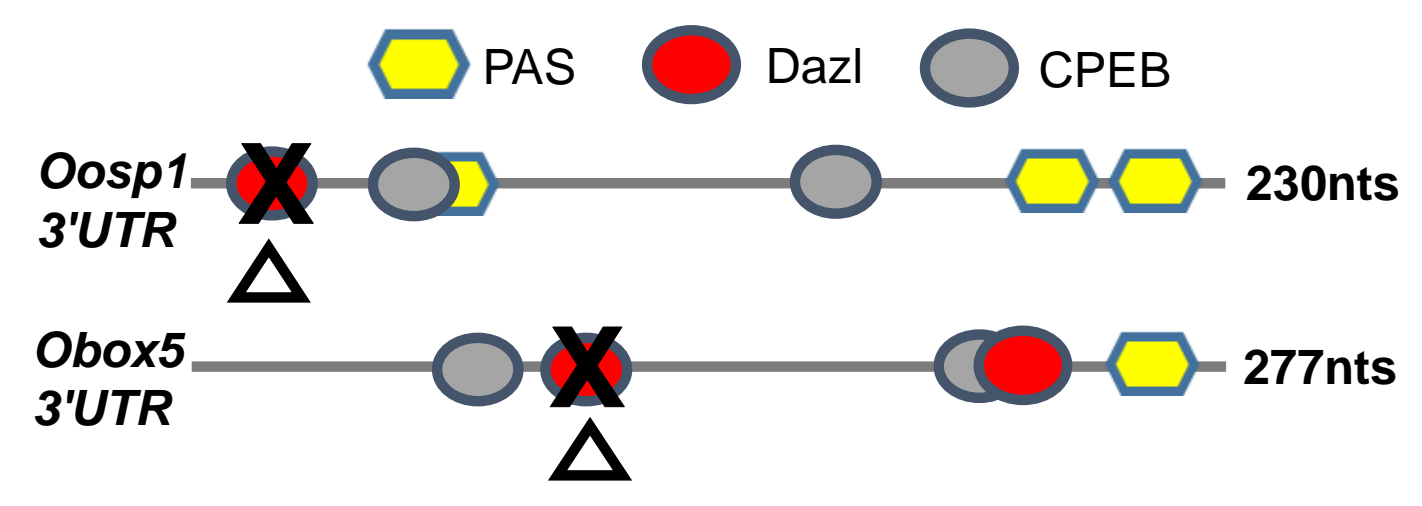

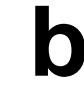

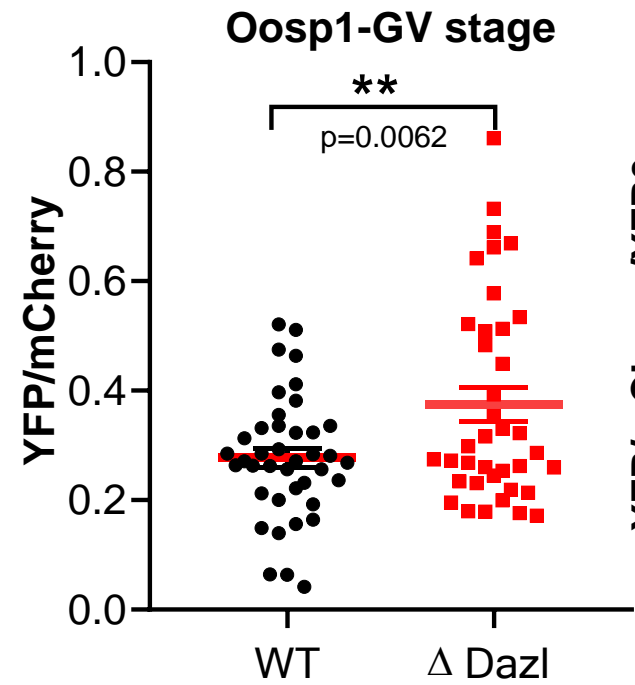

e

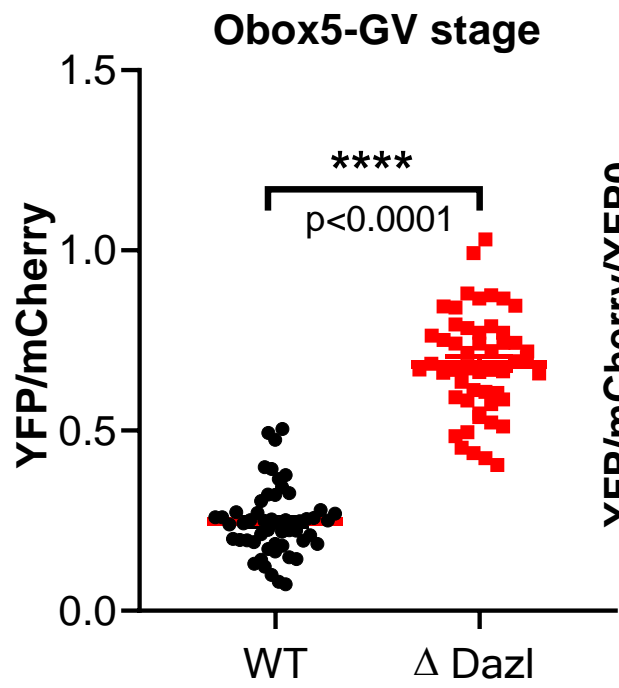

C

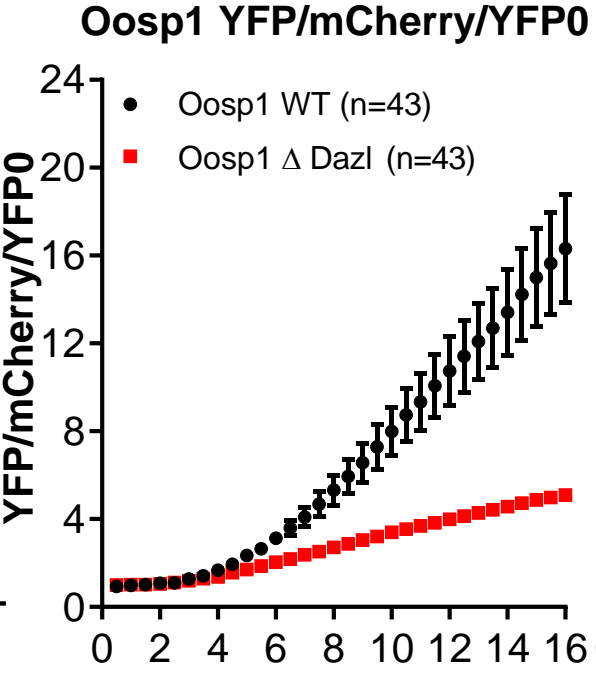

f

Obox5 YFP/mCherry/YFPO

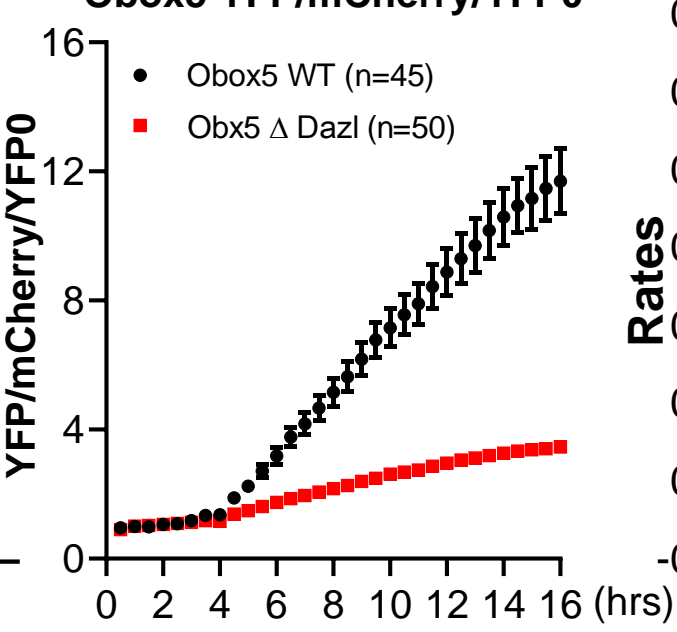

Rates

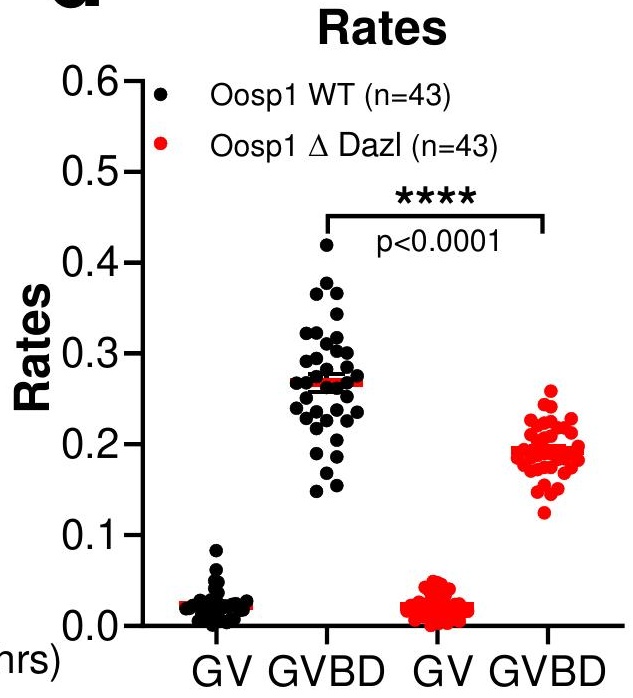

d 